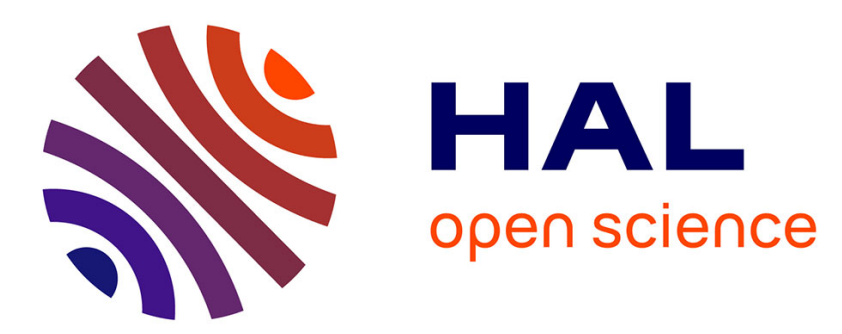

\title{
An Energy-Based Approach for n-dof Passive Dual-user Haptic Training Systems
}

Fei Liu, Angel Ricardo Licona Rodriguez, Arnaud Lelevé, Damien Eberard, Minh Tu Pham, Tanneguy Redarce

\section{- To cite this version:}

Fei Liu, Angel Ricardo Licona Rodriguez, Arnaud Lelevé, Damien Eberard, Minh Tu Pham, et al.. An Energy-Based Approach for n-dof Passive Dual-user Haptic Training Systems. Robotica, 2019, pp.on line. 10.1017/S0263574719001309 . hal-02176256

\section{HAL Id: hal-02176256 \\ https://hal.science/hal-02176256}

Submitted on 30 Aug 2019

HAL is a multi-disciplinary open access archive for the deposit and dissemination of scientific research documents, whether they are published or not. The documents may come from teaching and research institutions in France or abroad, or from public or private research centers.
L'archive ouverte pluridisciplinaire HAL, est destinée au dépôt et à la diffusion de documents scientifiques de niveau recherche, publiés ou non, émanant des établissements d'enseignement et de recherche français ou étrangers, des laboratoires publics ou privés. 


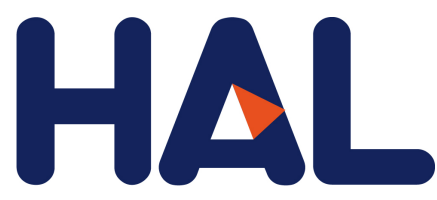

archives-ouvertes

\section{An Energy-Based Approach for n-dof Passive Dual-user Haptic Training Systems}

Fei Liu, Angel Licona Rodriguez, Arnaud Lelevé, Damien Eberard, Minh Tu Pham, Tanneguy Redarce

\section{To cite this version:}

Fei Liu, Angel Licona Rodriguez, Arnaud Lelevé, Damien Eberard, Minh Tu Pham, et al.. An Energy-Based Approach for n-dof Passive Dual-user Haptic Training Systems. Robotica, Cambridge University Press, In press, 10.1017/S0263574719001309 . hal-02176256

\section{HAL Id: hal-02176256 \\ https://hal.archives-ouvertes.fr/hal-02176256}

Submitted on 30 Aug 2019

HAL is a multi-disciplinary open access archive for the deposit and dissemination of scientific research documents, whether they are published or not. The documents may come from teaching and research institutions in France or abroad, or from public or private research centers.
L'archive ouverte pluridisciplinaire HAL, est destinée au dépôt et à la diffusion de documents scientifiques de niveau recherche, publiés ou non, émanant des établissements d'enseignement et de recherche français ou étrangers, des laboratoires publics ou privés. 


\section{An Energy-Based Approach for n-d.o.f. Passive Dual-user Haptic Training Systems Fei Liu†, Angel Ricardo Liconał, Arnaud Lelevéł*, Damien Eberard $\ddagger$ Minh Tu Pham $\ddagger$ and Tanneguy Redarce $\ddagger$}

$\dagger$ Center for Micro-BioRobotics IIT@SSSA, Italy $\ddagger$ Ampère (CNRS UMR 5005), Université de Lyon, INSA Lyon, F69621, Villeurbanne, France

(Accepted July 7, 2019. First published online: MONTH DAY, YEAR)

\section{SUMMARY}

This paper introduces a dual-user training system whose design is based on an energetic approach. This kind of system is useful for supervised hands-on training where a trainer interacts with a trainee through two haptic devices, in order to practice on a manual task performed on a virtual or teleoperated robot (for example for an MIS task in a surgical context). This paper details the proof of stability of an Energy Shared Control (ESC) architecture we previously introduced for one degree of freedom (d.o.f.) devices. An extension to multiple degrees of freedom is proposed, along with an enhanced version of the Adaptive Authority Adjustment (AAA) function. Experiments are carried out with 3 d.o.f. haptic devices in free motion as well as in contact contexts in order to show the relevance of this architecture.

KEYWORDS: Computer Simulation; Dual-user System; Hands-On Training; Haptic interfaces; Port-Hamiltonian Framework; Shared Control

\section{Introduction}

As in every profession where dexterous manipulation is necessary, medical staff require initial and continuing hands-on training on ever evolving medical methods. Medical simulators such as cadavers and animals have been a convenient way to learn by trial for decades in medical universities. However, due to the growing cost of providing them, noncontinuous availability problems and ethical issues, phantoms are increasingly used. Yet, phantoms provide a limited set of common cases to practice on. Providing a larger set of typical medical cases requires ownership of a great variety of available phantoms, which becomes rapidly costly. Nowadays, the over-crowded medical student populations are still offered too few opportunities to perform hands-on training during their curriculum, due to a limited access to hands-on training resources. For instance, epidural anesthesia requires much practice to be mastered enough to practice it on a real patient. A study ${ }^{28}$ by Vaughan et al. reported that it requires at least 80 anesthesia to reach a $90 \%$ success rate. Not so many trials are performed during students' studies. Therefore, it is necessary to provide cost-efficient solutions they could use during supervised sessions but also autonomously in order to sufficiently train themselves during their curriculum.

Over the last decade, Virtual Reality (VR) simulators $\left(\mathrm{see}^{29}\right.$ for a recent review on this topic) have been designed to overcome the drawbacks of aforementioned models. With virtual patient models which can be parameterized on-line, it becomes possible to provide an infinite set of medical cases with various difficulty levels.VR simulators have been progressively improved to provide to trainees a more realistic environment in

\footnotetext{
* Corresponding author. E-mail: arnaud.leleve@insa-lyon.fr
} 
$2 \mathrm{D}$ and more recently in 3D (with one example ${ }^{4}$ ). With haptic training simulators, the additional force feedback provides a realistic tool behavior, which leads to an efficient training for advanced tasks. ${ }^{17}$ More recently, the interest of haptic feedback in roboticassisted surgery has been proven by Talasaz et al. in the case of suturing application. ${ }^{26}$ It is demonstrated that force feedback plays a key role in helping users to manipulate the instruments better and to reduce tissue stress, tissue damage and accidental hits. These systems feature a haptic interface (i.e. a device by which tool-environment interaction forces are transmitted back to a human operator based on their hand motion) which acts as a master and a software architecture which connects the master to the slave. The slave is generally a software simulating a virtual tool inside a virtual environment.

Even if some simulators usually provide solutions to autonomously train oneself, for some difficult cases, it remains useful that a trainer guides the trainees' motions for a more accurate and efficient training. A trainer can directly guide the hands of a trainee to perform a correct motion but this "four hand fellowship" does not permit for the trainee to feel and dose the correct level of force to apply on their device in case of interaction of their tool with its environment. Dual-user systems are a practicable solution to this problem, as they can reproduce this important force information to both users, each one interacting with their own haptic interface. These systems extend the aforementioned master-slave architecture by adding a second master, as shown in Figure 1. The common concept is that the users share the slave control according to a dominance factor $(\alpha \in[0,1])$. When $\alpha=1$ (respectively 0 ), the trainer (respectively trainee) has full authority on the slave and on the trainee's (respectively trainer's) device. When $0<\alpha<1$, both users share the slave control with a dominance (over the other user) which is function of $\alpha$. According to the architectures found in the literature, the effect of $\alpha$ on the force feedback provided to the users differs. These differences are highlighted in section 2 .

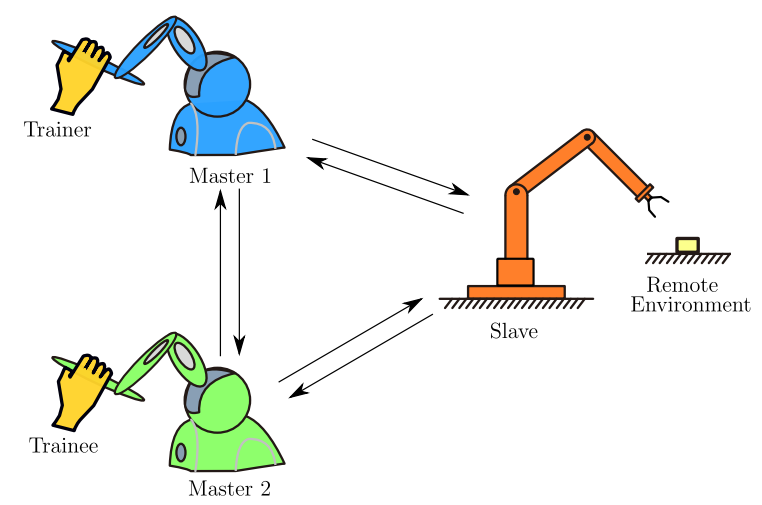

Fig. 1. Architecture of a dual-user system

The objective of the current project is to design a dual-user hands-on haptic training system. In this system, trainer and trainee manipulate their own haptic interface. They share the same "slave" which can be either a real surgical robot dived into a real environment, located in a near black box for instance, or a virtual robot in a virtual environment, provided by a software simulation. Such an application, providing haptic mentoring, has been introduced by Chebbi et al. ${ }^{3}$ We focus on applications with two masters and one slave, all located in the same room. The models proposed hereafter will be extended in the future to cope with transport delay consequences on stability and transparency in order to permit a distant use.

The following typical use case helps determine the main requirements of the system. Suppose, at first, that the trainer (an experienced surgeon) aims at demonstrating the right trajectories of their surgical tool to perform a task featuring free motions and some tool-environment contacts. This implies that they require a realistic force feedback to dose their force, as in a bilateral teleoperation context. The trainer manually sets $\alpha=1$ to become leader (the trainee becomes follower): it is a mentoring mode. They then 
get a full force feedback from the slave in order to perform their task as if they were handling the real instruments. Meanwhile, the trainee's device follows the trajectory of the leader one. If the trainee deviates from this reference trajectory when in free motion, the compliance of the device brings them back to the right position. In case of interaction between the tool and its environment, the trainee can also feel in their hands the right level of effort to provide to the tool, by means of a display which guides them to set their device at the right position with the right applied force. Afterwards, the functioning can be inverted by reversing $\alpha$ so that the trainee manipulates and the trainer follows and evaluates trainee's motions and applied forces: it is an evaluation mode.

The analysis of the scientific literature showed that the existing solutions did not fit all the requirements of such a use case. Reasons are detailed in section 2. Thus, a dual-user control architecture had been previously introduced: the Energy based Shared Control (ESC). ${ }^{12}$ It reuses the robust control approach introduced by Stramigioli, ${ }^{25}$ which ensures passivity even in presence of robot model uncertainties, limited bandwidth, or non-linearities (saturations). It thus provides robust passive and compliant interfaces. In Liu et al. ${ }^{12}$ ESC was developed for single degree of freedom (d.o.f.) mechanisms, with experiments conducted with only two real haptic devices and a virtual one. We used an energetic approach which guarantees the passivity of the system whatever evolution of $\alpha$, which is not always proven in existing frameworks (in LTI models, $\alpha$ is supposed to be constant or sufficiently slowly evolving to consider it as a constant in the models). We then proposed to embed an Adaptive Authority Adjustment (AAA) function to automatically revert, in evaluation mode, the authority from the trainee to the trainer in case of bad gestures performed by the trainee. ${ }^{11}$ However, this solution required to tune two task-dependent parameters which were hard to be optimized by any trainer. ESC has been compared with two other dual-user architectures from Khademian and Hashtrudi-Zaad (the "Complementary Linear Combination" (CLC) and the "Masters Correspondence with Environment Transfer" (MCET)), by means of simulations. ${ }^{13}$ This comparative study concludes, from simulation results, that the ESC has position tracking performance equivalent to the one of CLC and MCET architectures. Yet, ESC force tracking performance is intrinsically better for hands on training purpose as the former architectures cannot perform mentoring and evaluation involving simultaneous force and position tracking for both users. We also enhanced the ESC (where the environment and users were assumed as passive) by introducing a Time Domain Passivity Controller (TDPC) to keep the system passive whatever user or environment and whatever value of $\alpha$ and every parameter tuning the system compliance. ${ }^{13}$ More recently, we reported some preliminary experiments testing the opportunity to extend this approach to several degrees of freedom. ${ }^{10}$ As this provided encouraging results, we introduce here this novelty.

Compared to these former publications, we introduce in this new paper the following contributions:

- The Port Hamiltonian modeling of this architecture, only evoked in previous papers, is detailed.

- The extension to multiple degrees of freedom for haptic devices with same kinematics is discussed, which makes this architecture usable in practice in many cases, as the both users should use same device types.

- The proof of passivity is extended to $n$-d.o.f. systems, with the help of an updated TDPC.

- The Adaptive Authority Adjustment (AAA) is extended to $n$-d.o.f. and also enhanced: it henceforth requires only one threshold parameter which can be tuned on-line by the trainer, without specific skill.

Real-time experiments are performed with a 3-d.o.f. architecture and three real haptic devices in order to validate these enhancements. They are shown in user (cartesian) space for the sake of clarity.

This paper is organized as follows. Section 2 describes the evolution of the dual-user concept in the literature. Section 3 details the ESC based dual-user system model. Section 4 proves the passivity of the architecture with several d.o.f. along with an 
Table I . Nomenclature.

\begin{tabular}{lll}
\hline Notation & \multicolumn{1}{c}{ Meaning $(i \in\{1,2\})$} & Unit \\
\hline$\alpha$ & Main dominance factor & \\
$\beta_{1}, \beta_{2}$ & ASM internal energy distribution factors & \\
$\left(\dot{\theta}_{m_{i}}, t_{h_{i}}\right)$ & User $i$ - Master $i$ power port & $(\mathrm{rad} / \mathrm{s}, \mathrm{N} . \mathrm{m})$ \\
$\left(\dot{\theta}_{m_{i}}, t_{m_{i}}\right)$ & Master $i$ - IPC M $i$ power port & $(\mathrm{rad} / \mathrm{s}, \mathrm{N} . \mathrm{m})$ \\
$\left(\dot{\theta}_{r_{i}}, t_{r_{i}}\right)$ & IPC M $i$ - ASM power port & $(\mathrm{rad} / \mathrm{s}, \mathrm{N} . \mathrm{m})$ \\
$\left(\dot{\theta}_{r_{s}}, t_{r_{s}}\right)$ & ASM power port - IPC S power port & $(\mathrm{rad} / \mathrm{s}, \mathrm{N} . \mathrm{m})$ \\
$\left(\dot{\theta}_{s}, t_{s}\right)$ & IPC S - Slave power port & $(\mathrm{rad} / \mathrm{s}, \mathrm{N} . \mathrm{m})$ \\
$\left(\dot{\theta}_{s}, t_{e}\right)$ & Slave - Environment power port & $(\mathrm{rad} / \mathrm{s}, \mathrm{N} . \mathrm{m})$ \\
\hline
\end{tabular}

improved version of the Adaptive Authority Adjustment (AAA) mechanism. Section 5 provides experimental results to demonstrate the relevance of the aforementioned developments.

\section{Existing Dual-user Architectures}

Various architectures have been proposed for the control of dual-user haptic systems. The "shared control" concept has been introduced by Nudehi et al. in ${ }^{16}$ for Minimal Invasive Telesurgical Training purpose, i.e. to "allow experienced surgeons mentor trainee surgeons through shared control of a surgical robot". The control of the slave is shared between both users, and each one experiences feedback forces proportional to the difference of position of their own device, and to $\alpha$. In any case, an interaction between the robot and its environment would not feed back interaction forces to any user. Therefore, this kind of training system can only be used to train users on motions, not on efforts.

Khademian and Hashtrudi-Zaad overcame this limitation by providing a three-port multilateral control architecture. ${ }^{7}$ Two distinct architectures are proposed by the authors. The first one is the Complementary Linear Combination (CLC) architecture which provides feedback forces combining the environment and the other user forces (typically $F_{e}$ and $F_{h_{2}}$, for user 1). The desired position and force commands for each device are a complementary weighted sum of positions and forces of the other two devices. When $\alpha=1$, master 1 and the slave form a four-channel bilateral teleoperation system. The position of the device 1 and the forces the user 1 applies on it, also serve as input for the master 2 controller (the behavior is symmetric when $\alpha=0$ ). When $0<\alpha<1$ both users can perform a task collaboratively in a shared environment. On initial examination, this architecture is the solution which best fits this project's requirements. Unfortunately, when trying to perform a mentoring (respectively evaluation) session, the trainee (respectively trainer) has no direct force feedback from the environment, only from the other user. This is why the authors advise to use this system with values of $\alpha=0.2,0.5,0.8$ to get the best transparency. Yet, in these cases, both users influence the slave trajectory, which may lead from inaccurate to trembling motions, not compliant with mentoring and evaluation modes. The second architecture Khademian and Hashtrudi-Zaad introduced is called "Masters Correspondence with Environment Transfer" (MCET), ${ }^{7}$ where both user devices follow the motion of each other and the effort fed back to both users is $F_{e} / 2$. The command to the slave is weighted accordingly by $\alpha$ and $(1-\alpha)$. Kinesthetic performance analysis and experimental user perception studies performed by these authors showed that the MCET architecture provides a better kinesthetic feedback to both users with the least sensitivity to the dominance factor, compared with CLC. Yet, still according to the authors, this architecture transmits a distorted environment force to both users (which was equal to $F_{e} / 2$ in addition with a force expressed as a function of the difference of the positions of the devices of both users), which does not comply with the aforementioned use-case.

Ghorbanian et al. defined two dominance factors $\alpha$ and $\beta .^{6} \alpha$ determines the balance of authority between the trainer and the trainee, while $\beta$ indicates the supremacy of 
both trainer and trainee over the slave robot. This provides an additional degree of freedom in the authority mechanism which is used to set a nonlinear relation between $\alpha$ and $\beta$ in order to adjust the authority of the leader (the user for which $\alpha>0.5$ ) over the slave with respect to their authority over the follower. It also supports uncertain communication channels in presence of delays, packet loss, data duplication and packet swapping, which corresponds to practical issues to overcome as soon as masters and slave are away. However, when $\alpha=1$, the desired Master 2 force $\left(F_{h 2_{d}}\right)$ is only linked to the position of Master 1, not to the tool-environment interaction force, which does not fit our training requirements either. Also, as this work is not compared with CLC or MCET, it cannot be determined whether the transparency of this architecture is better.

The architecture introduced by Razi et al. ${ }^{19}$ transmits to the follower a force $F_{h 2_{d}}=$ $\left(F_{e}-\alpha F_{h_{1}}\right)$, where $F_{h_{1}}$ is the force applied by user 1 on their device, which also does not correspond to this project requirements. An interesting work has been introduced by Shamaei et $a l . .^{23}$ where two dominance factors are used. Several architectures are provided. The one which tracks the positions of all three devices is experimentally evaluated. This architecture's performance is analyzed in terms of impedance matching between trainer and trainee during soft and hard interactions. This provides interesting but partial information during interaction: no information about position nor force absolute tracking error is provided, only a comparison versus the two dominance factors. Also, the authors specify that this approach is limited in terms of control freedom as derivative operators may destabilize it.

In ${ }^{22}$ the users' haptic feedback is a weighted (with $\alpha$ ) sum of the virtual toolenvironment interaction force $F_{e}$ and the other user interaction (with their master device) force $F_{h}$. More precisely, the desired haptic feedback applied to user 1 is $F_{h_{1} d}=\alpha . F_{e}+$ $(1-\alpha) F_{h_{2}}$. Thus, when $\alpha=1$, user 1 only feels $F_{e}$ and user 2 only feels $F_{h_{1}}$. Experiments show that when $\alpha=0.5$ and both users follow very close trajectories, they feel very close force feedback, in presence of time delays between each master and the slave. This is an interesting result. Yet, both users must kind of cooperate to guide the slave tool with the same dominance. In our point of view, it may disturb the mentoring/evaluation as each user are implied in the motions and the interactions without distinction. Also the experiments have been performed with constant environment interaction characteristics. More realistic experiments should mix free motion and interaction phases in the same session.

Furthermore, in a dual-user hands-on training system, the authority factor $\alpha$ should be switched anytime by the trainer. The architectures should guarantee that fast changes of $\alpha$ may not destabilize the system. Yet CLC, MCET and Razi et al. architectures have been modeled as LTI systems. Therefore, the robustness of these architectures should be evaluated according to the varying parameter $\alpha$. This has not been ensured yet as far as we know. As far as we could observe, the only architectures for which robustness versus $\alpha$ variations is proven are the ones in ${ }^{6}$ and,$^{22}$ through a Lyapunov function, and the one from Shamaei et al. ${ }^{23}$ through an unconditional stability approach based on the Llewellyn's criterion.

In 2011, a survey ${ }^{15}$ has been conducted concerning passivity-based controllers for nonlinear bilateral teleoperation. In our point of view, an energy approach seems promising as it already provided interesting results in more classical teleoperation studies (with two examples ${ }^{8}{ }^{9}$ ). Since this work has been realized, Lu et al. proposed to use three dominance factors to provide more flexibility to the users. ${ }^{14}$ These factors are dynamically adapted by extending the Online Authority Adjustment (OAA) mechanism (introduced in ${ }^{12}$ ), in the presence of communication delays. They use a linear modeling to prove the stability and study the transparency of the system. Also, Zakerimanesh et al. proposed to consider the actuator saturations ${ }^{30}$ (they only validated this proposal in simulation so far).

This review shows that despite of advanced works in this topic, none fully complies with the aforementioned use-case. The equivalent performance of the ESC compared to CLC and MCET architectures, established in simulation ${ }^{13}$ motivates to further work on this approach. 


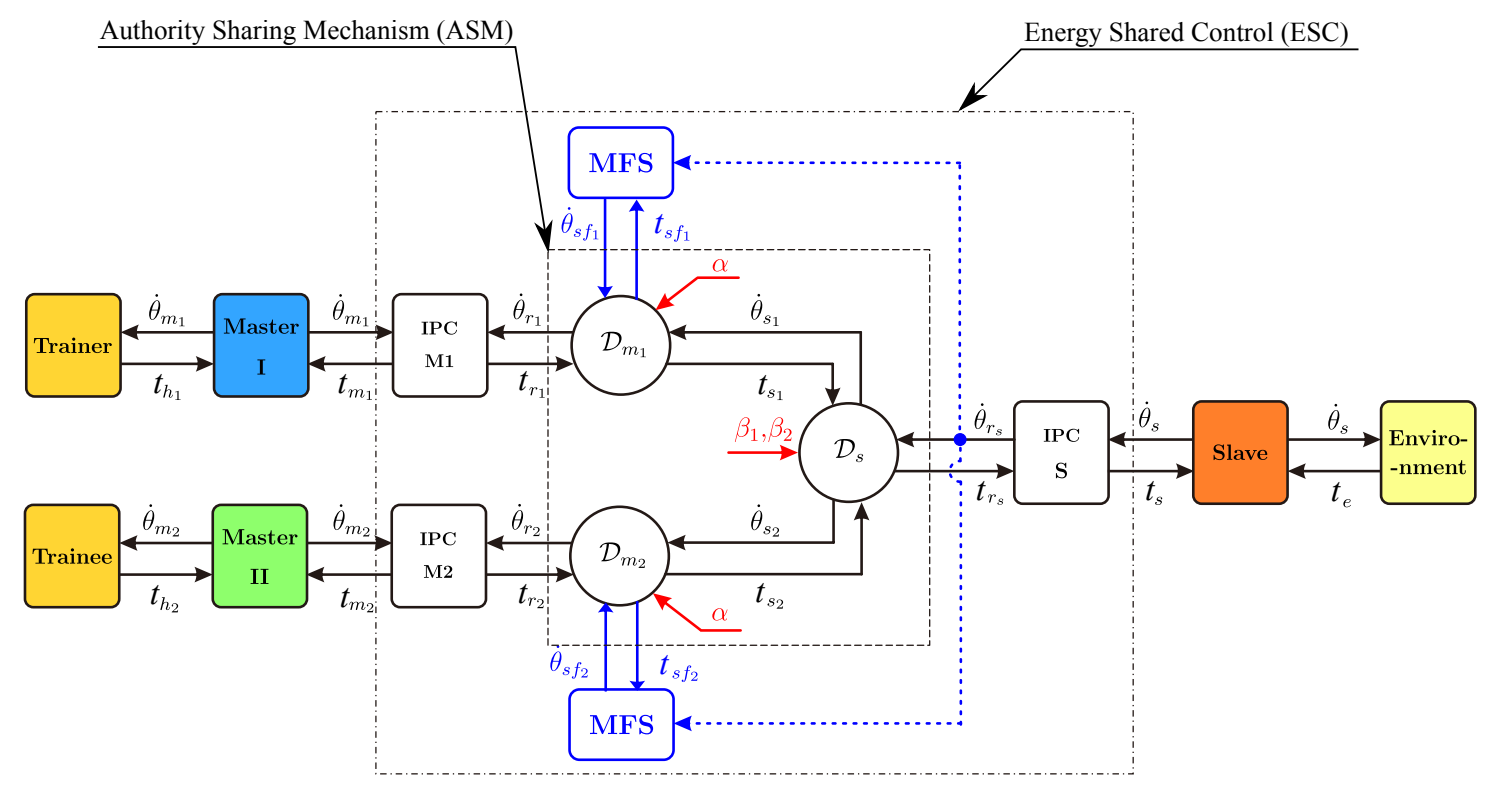

Fig. 2. Energy Shared Control (ESC) Dual-user system connected to haptic devices in attendance of both users and an environment (the authority sharing mechanism is located inside the inner dashed box).

\section{Energy Shared Control Based Dual-User Architecture}

This section details the design of a passive dual-user architecture, using an energetic approach. The scalability of this approach is a clear advantage: adding passive parts to a passive network, with lossless (and therefore passive) interconnections preserves the passivity. Thus, extending a teleoperation system with a second or more users is practicable with a moderated increase of model complexity. The design of this architecture started from a dual-user intrinsically passive system detailed in subsections 3.1 and 3.2. Yet, as this architecture was not able to provide full slave feedback to both users, it has been enhanced (see subsection 3.3).

\subsection{Authority Sharing Mechanism}

Inspired by the CLC architecture, ${ }^{7}$ we proposed an Authority Sharing Mechanism (ASM) by considering an energetic approach, as shown in Figure 2 (both "MFS" blocks can be ignored as of now; they will be introduced in the next subsection). The ASM, based on parameter $\alpha$, is located in the inner dashed box. It is composed of three linear Multiple-Input-Multiple-Output (MIMO) subsystems $\mathcal{D}_{m_{1}}, \mathcal{D}_{m_{2}}$ and $\mathcal{D}_{s}$ which behavior is introduced in equations (1), (2) and $(3)^{1}$. They, all together, redirect the energy flows (composed of angular velocity and torque signals) exchanged between the trainer device $\left(\dot{\theta}_{r_{1}}, t_{r_{1}}\right)$, the trainee's one $\left(\dot{\theta}_{r_{2}}, t_{r_{2}}\right)$ and the slave $\left(\dot{\theta}_{s}, t_{s}\right)$. The two additional power ports $\left(\dot{\theta}_{s f_{1}}, t_{s f_{1}}\right),\left(\dot{\theta}_{s f_{2}}, t_{s f_{2}}\right)$, and the effective role of $\mathcal{D}_{m_{1}}$ and $\mathcal{D}_{m_{2}}$ are introduced in next subsection. $\left(\dot{\theta}_{s f_{1}}, t_{s f_{1}}\right)$ and $\left(\dot{\theta}_{s f_{2}}, t_{s f_{2}}\right)$ can be considered null as of now. Three dominance factors $\alpha, \beta_{1}, \beta_{2} \in[0,1]$ are introduced to achieve the goal of shared control.

\footnotetext{
1 The notations of time-varying variables with $*(t)$ are abridged for the sake of clarity.
} 


$$
\begin{array}{lll}
\mathcal{D}_{m_{1}}:\left(\begin{array}{c}
\dot{\theta}_{r_{1}} \\
t_{s_{1}} \\
t_{s f_{1}}
\end{array}\right) & =\left(\begin{array}{ccc}
0 & \alpha(1-\alpha) \\
-\alpha & 0 & 0 \\
(\alpha-1) & 0 & 0
\end{array}\right) & \left(\begin{array}{c}
t_{r_{1}} \\
\dot{\theta}_{s_{1}} \\
\dot{\theta}_{s f_{1}}
\end{array}\right) \\
\mathcal{D}_{m_{2}}:\left(\begin{array}{c}
\dot{\theta}_{r_{2}} \\
t_{s_{2}} \\
t_{s f_{2}}
\end{array}\right) & =\left(\begin{array}{ccc}
0 & (1-\alpha) \alpha \\
(\alpha-1) & 0 & 0 \\
-\alpha & 0 & 0
\end{array}\right) & \left(\begin{array}{c}
t_{r_{2}} \\
\dot{\theta}_{s_{2}} \\
\dot{\theta}_{s f_{2}}
\end{array}\right) \\
\mathcal{D}_{s}:\left(\begin{array}{c}
\dot{\theta}_{s_{1}} \\
\dot{\theta}_{s_{2}} \\
t_{r_{s}}
\end{array}\right) & =\left(\begin{array}{ccc}
0 & 0 & \beta_{1} \\
0 & 0 & \left(1-\beta_{2}\right) \\
\beta_{1}\left(1-\beta_{2}\right) & 0
\end{array}\right) & \left(\begin{array}{c}
t_{s_{1}} \\
t_{s_{2}} \\
\dot{\theta}_{r_{s}}
\end{array}\right)
\end{array}
$$

The relationships between $\alpha, \beta_{1}$ and $\beta_{2}$ are defined as,

$$
\beta_{1}=\left\{\begin{array}{ll}
0, & \alpha=0 \\
1, & \alpha>0
\end{array} \quad \beta_{2}= \begin{cases}1, & \alpha=1 \\
0, & \alpha<1\end{cases}\right.
$$

The introduction of $\beta_{1}$ and $\beta_{2}$ provides a nonlinear solution to avoid the repetition of the authority distribution in $\mathcal{D}_{s}$ (which would provoke some port relations embedding $\alpha^{2}$ terms, which was not desired), since the shared power flow is already formed at $\mathcal{D}_{m_{1}}$ and $\mathcal{D}_{m 2}$. The role of $\mathcal{D}_{s}$ is to sum the torques from the masters to the slave and deliver the velocities to both users. We obtain three modes by changing the dominance factor $\alpha$ : mentoring mode $(\alpha=1)$, guidance mode $(0<\alpha<1)$ and evaluation mode $(\alpha=0)$.

The three nodes $\left(\mathcal{D}_{m_{1}}, \mathcal{D}_{m_{2}}\right.$ and $\left.\mathcal{D}_{s}\right)$ form a power-conserving interconnection. This means that the global authority sharing mechanism is passive and lossless. This is represented by the following power balance:

$$
t_{r_{1}}^{T} \dot{\theta}_{r_{1}}+t_{r_{2}}^{T} \dot{\theta}_{r_{2}}+t_{r_{s}}^{T} \dot{\theta}_{r_{s}}+\left(t_{s f_{1}}^{T} \dot{\theta}_{s f_{1}}+t_{s f_{2}}^{T} \dot{\theta}_{s f_{2}}\right)=0
$$

which indicates the ASM passivity.

\subsection{Haptic Interface Control}

ASM does only redirect signals between the three power ports, it does not permit an efficient control of the end interfaces (Master 1, Master 2 and Slave). For this purpose, we inserted three Intrinsically Passive Controllers (IPC) (respectively IPC M1, IPC M2 and IPC S) in between them (see Appendix). The IPC controllers, introduced by Stramigioli ${ }^{25}$ have the property to ensure a robust passive interaction of the controlled system with an unknown environment, without having to model both ones. As the three interconnected devices (Master 1,2 and Slave) are interacting respectively with users and any environment, IPC controllers are interesting solutions for the three of them. Therefore, by linking them to the ASM architecture through power-conserving interconnections, we obtain a new passive system, whatever controllers' parameters, devices', users' and environment's characteristics. This ensures a structural robustness and leaves freedom to tune every IPC parameter for performance and global transparency. Moreover, IPC controllers add some compliance which helps in manipulation and training tasks.

\subsection{Providing Force and Position Feedback to Both Users}

This ASM architecture has a defect when $\alpha=1$ (respectively 0 ): the trainer (respectively trainee) exchanges all their energy exclusively with the slave. The system acts this way as a classical teleoperation system and completely isolates the trainee (respectively trainer). The latter cannot control the slave, which is desired, but they also do not get any feedback from it, which is opposed to the aforementioned training requirements. In order to solve 
this problem, we added two identical Modulated Flow Sources (MFS) visible in Figure 2 which role is to "copy" $\dot{\theta}_{r_{s}}$ coming from the slave IPC without directly exchanging any energy. The aim of these two flow sources is to feed back the complementary motion information from the slave which is missing in the ASM architecture. Consequently, both users experience a full motion feedback regardless of the control authority (i.e. the value of $\alpha$ ). This is checked by introducing $\dot{\theta}_{s f_{i}}=\dot{\theta}_{r_{s}}(i \in\{1 ; 2\})$ into Eq. (1) and (2):

$$
\begin{aligned}
& \dot{\theta}_{r_{1}}=\alpha \dot{\theta}_{s_{1}}+(1-\alpha) \dot{\theta}_{s f_{1}}=\alpha \dot{\theta}_{r_{s}}+(1-\alpha) \dot{\theta}_{r_{s}}=\dot{\theta}_{r_{s}} \\
& \dot{\theta}_{r_{2}}=(1-\alpha) \dot{\theta}_{s_{2}}+\alpha \dot{\theta}_{s f_{2}}=(1-\alpha) \dot{\theta}_{r_{s}}+\alpha \dot{\theta}_{r_{s}}=\dot{\theta}_{r_{s}}
\end{aligned}
$$

It is worth mentioning that additional energy may be injected into the system by the modulated flow sources. This could violate the passivity of the whole architecture which may cause unstable behaviors. This aspect is discussed in section 4 .

Remark: This way, thanks to the IPC controller properties (see section 3.2 and Appendix), for example, when $\alpha=0, t_{h_{2}}$ tends towards $t_{e}$ but also $t_{h_{1}}$ tends towards $t_{e}$ when the trainer moves their master interface such a way that $\theta_{m_{1}} \rightarrow \theta_{m_{2}}$. One can see an illustration of it in the plots of the experimental results in Section 5 .

Thus, all together, we obtain an intrinsically passive dual-user system, named "Energy Shared Control" (ESC), which corresponds to the outer dash-dot box in Figure 2.

\subsection{Extension to Multiple Degrees of freedom}

As the training applications with only one d.o.f. are limited, we applied the ESC architecture to systems with multiple degrees of freedom. As a first approach, we deal with training systems having master and slave devices with same kinematics. This is not a strong assumption as for a given training application, master devices should be identical. Using the same device model is also coherent. Concerning the slave robot, this assumption is a little more restricting as it should be more practical to use a dedicated robot to handle some tools. The aforementioned one d.o.f. architecture has been expanded to $n$ d.o.f. by duplicating the previous one-d.o.f. architecture independently for each joint (see Figure 3). We assumed that the potential interactions between each d.o.f. would naturally balance each other as we use same device kinematics and that the robustness of the IPC controllers would absorb potential disturbances. In future works, we will deal with interfaces with different kinematics.

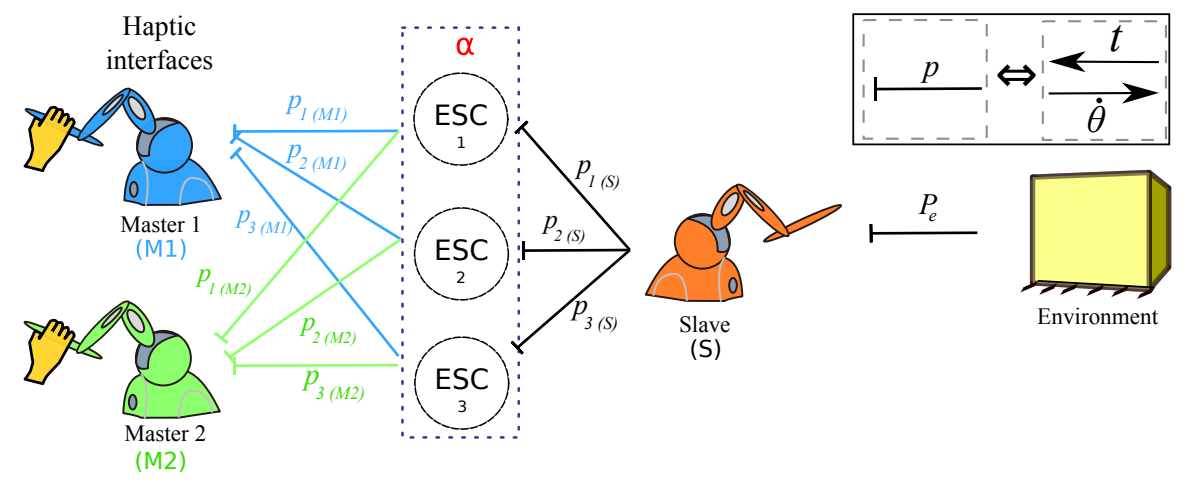

Fig. 3. Multiple d.o.f. articular ESC architecture, here for three joints

\subsection{Adaptive Authority Adjustment (AAA) Enhancement}

The Adaptive Authority Adjustment (AAA) function, ${ }^{11}$ permits the automatic adjustment of $\alpha$ in real time during an evaluation. In evaluation mode, the trainee is leader by default but when the trainer voluntarily deviates from the trainee's trajectory (potentially because of an error performed by the trainee), the trainer automatically 
gets back the authority over the slave. When the trainee's device comes back close to the position of the trainers' one, the authority is automatically set back to the trainee.

We propose here to improve the previous solution which was introduced for a one d.o.f. system. This new algorithm applies for $n$ d.o.f. systems and now requires only one parameter adjustable in real time by the trainer (versus two parameters which were hard to tune in the previous proposal): $e_{\max }$ which can be tuned on-line by the trainer according to the desired strictness during the evaluation. It is based on a Bézier polynomial proposed by Beltran-Carbajal in another context, ${ }^{2}$ which sets $\alpha$ (the same value for every joint) in real time, as a smooth function (see Figure 4 and Eq. (7)) of the deviation of position between the master devices $e(t)$ :

$$
\alpha(t)=\left\{\begin{array}{cc}
e_{r}(t)^{5}\left[r_{1}-r_{2} e_{r}(t)+r_{3} e_{r}(t)^{2}-\right. & e_{r}(t) \leq 1 \\
\left.r_{4} e_{r}(t)^{3}+r_{5} e_{r}(t)^{4}-r_{6} e_{r}(t)^{5}\right] & e_{r}(t)>1 \\
1 &
\end{array}\right.
$$

where $e_{r}(t)=\frac{e(t)}{e_{\max }}$ is the relative error, $e(t)=\sqrt{\sum_{1}^{n} e_{i}(t)^{2}}$ is the global magnitude of the instantaneous articular errors $e_{i}(t)=\left|\theta_{m_{1 i}}(t)-\theta_{m_{2} i}(t)\right|$ with $i \in\{1 . . n\}$, and $e_{\max }$ is the deviation which implies a return to full authority of the trainer over the slave. $r_{1}=252, r_{2}=1050, r_{3}=1800, r_{4}=1575, r_{5}=700$ and $r_{6}=126$. In practice, it has not been necessary to compute a mean error on a floating time frame.

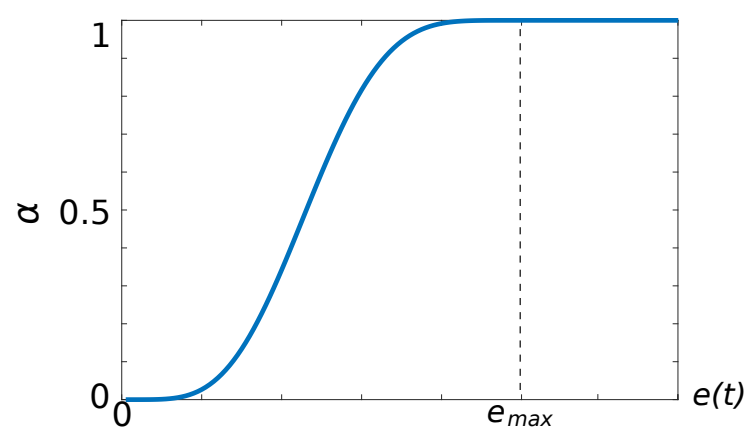

Fig. 4. Bézier based function to adapt $\alpha$ according to the position deviation between master devices

Remark: Section 5.4 shows how the system behaves in practice. This is a simple but efficient solution. A more evolved approach has been proposed by Shahbazi et $a l .{ }^{21}$ with the help of fuzzy logic (FL). This approach cannot be directly used with our architecture as the FL simultaneously tunes online two dominance factors as in Ghorbanian's architecture. ${ }^{6}$ A modification of the ASM part would be necessary to ensure that it remains passive whatever dominance values of $\alpha$.

\section{Proof of Passivity}

In this section, the passivity condition is evaluated. To do so, the system is modeled using the port-Hamiltonian approach ${ }^{27}$ as it provides a compact model of the form of Eq. (8). A passivity controller is then proposed to ensure a stable behavior.

\subsection{Global Model}

In the following, uppercase variables signify vectors of $n$ variables with the same name for each joint (typ. $\left.A=\left(a_{1} a_{2} \ldots a_{n}\right)^{T}\right)$. The energetic model of the whole system including the ESC and the haptic devices can be expressed as: 


$$
\left\{\begin{array}{l}
\dot{X}=[J-R(X)] \frac{\partial H}{\partial X}+G U+Q V \\
Y=G^{T} \frac{\partial H}{\partial X} \\
S=Q^{T} \frac{\partial H}{\partial X}
\end{array}\right.
$$

where the state variables are

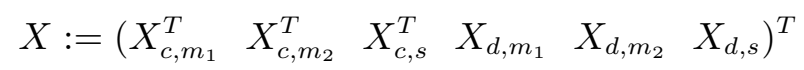

with $X_{c, i}$ (for $i \in\left\{m_{1}, m_{2}, s\right\}$ ) being the state variables of each IPC controller duplicated for each d.o.f., and $X_{d, i}$ the state variables of each haptic device (see Appendix).

$J$ is a skew-symmetric matrix which models the internal state variable interconnections. $R(X)$ is a matrix which represents the internal dissipation. $H(X)$ is the global system Hamiltonian.

The input $U=\left(\begin{array}{lll}T_{h_{1}} & T_{h_{2}} & T_{e}\end{array}\right)^{T}$ (of size $n \times 3$ ) is composed of the external torques and $G$ is a matrix which describes how these torques are distributed into the system.

$Y=\left(\begin{array}{lll}\dot{\Theta}_{m 1} & \dot{\Theta}_{m 2} & \dot{\Theta}_{s}\end{array}\right)^{T}$ is the angular velocity output (of size $\left.n \times 3\right)$. The input $V=\left(\begin{array}{ll}\dot{\Theta}_{s f 1} & \dot{\Theta}_{s f 2}\end{array}\right)^{T}$ (of size $\left.n \times 2\right)$ represents the virtual velocities provided by both MSF and $S=\left(\begin{array}{lll}T_{s f 1} & T_{s f 2}\end{array}\right)^{T}$ (of size $\left.n \times 2\right)$ is composed of the virtual output torques sent to the MSF.

This model is built by lossless interconnection of subsystems which can be modeled each one in a port hamiltonian way. The only light assumption is that the haptic devices can be modeled this way, as proposed in. ${ }^{5}$ The energetic ports (angular velocity - torque) of the environment, users (trainer and trainee), master and slave devices, IPC master and slave controllers and the ESC are all interconnected following the Figure 2. See the Appendix concerning the definition of ports of devices and IPC controllers.

\subsection{System Passivity}

4.2.1. ESC Passivity Analysis. The global system features both the human/environment power port $(U, Y)$ and the modulated source power port $(V, S)$. The energy balance is given by

or, equivalently:

$$
\frac{d H}{d t}=-\frac{\partial^{T} H}{\partial X} R \frac{\partial H}{\partial X}+Y^{T} U+S^{T} V
$$

$$
\begin{aligned}
& H(X(t))-H(X(0)) \\
& =-D(t)+\int_{0}^{t} Y^{T}(\tau) U(\tau) \mathrm{d} \tau+\int_{0}^{t} S^{T}(\tau) V(\tau) \mathrm{d} \tau
\end{aligned}
$$

where $D(t)$ is a non-negative function which represents the energy dissipated in the system.

Only the human/environment power port $(U, Y)$ is associated with the external world. Therefore, $H(X(t))-H(X(0))$ is a storage function if the following inequality holds:

$$
H(X(t))-H(X(0)) \leq \int_{0}^{t} Y^{T}(\tau) U(\tau) \mathrm{d} \tau
$$

As $D(t) \geq 0$, a sufficient condition to respect this property is to impose that : 


$$
\int_{0}^{t} S^{T}(\tau) V(\tau) \mathrm{d} \tau \leq 0
$$

From the leader user point of view (trainer in mentoring mode, trainee in evaluation mode), the system is composed of lossless interconnections of passive subsystems.

Otherwise, the two MFS could inject extra energy into the system and potentially destabilize it. By integrating Eq. (1) and (8) and knowing that $\dot{\theta}_{s f_{1}}=\dot{\theta}_{s f_{2}}=\dot{\theta}_{r_{s}}$, the previous passivity condition can be rewritten as:

$$
\begin{aligned}
E_{p}(t) & =E_{p}(0)+\int_{0}^{t} S^{T}(\tau) V(\tau) \mathrm{d} \tau \\
& =0+\int_{0}^{t}\left[T_{s f_{1}}^{T}(\tau) \dot{\Theta}_{s f_{1}}(\tau)+T_{s f_{2}}^{T}(\tau) \dot{\Theta}_{s f_{2}}(\tau)\right] \mathrm{d} \tau \\
& =\int_{0}^{t}\left[(\alpha-1) T_{r_{1}}(\tau)-\alpha T_{r_{2}}(\tau)\right]^{T} \dot{\Theta}_{r_{s}}(\tau) \mathrm{d} \tau
\end{aligned}
$$

So, the system will remain passive as long as:

$$
E_{p}(t) \leq 0 \quad \forall t \geq 0
$$

4.2.2. Passivity Controller. In practice, in order to respect the passivity condition of Eq. 14, we modulate the Modulated Flow Source outputs as indicated:

$$
\dot{\Theta}_{s f_{1}}=\dot{\Theta}_{s f_{2}}= \begin{cases}\dot{\Theta}_{r_{s}}, & \text { if } E_{p} \leq 0 \\ 0, & \text { otherwise }\end{cases}
$$

As long as the condition in Eq. 14 is satisfied, both MFS are activated, providing full feedback to both users (mainly to the follower one). Otherwise, both MFS are deactivated, forcing the modulated flows $\dot{\Theta}_{s f_{1}}$ and $\dot{\Theta}_{s f_{2}}$ to 0 . This passivity controller always guarantees the global passivity and only deteriorates the additional feedback in case of potential loss of passivity. This means that in critical situations, the quality of the feedback is downgraded to the one provided in section 3.1. The main drawback is that, in mentoring and evaluation modes, the follower user becomes isolated as long as the passivity controller triggers. Experiments did not permit us to encounter such a situation. This is why we did not look for a less conservative solution up to now. This could be a suggestion of improvement.

\section{Real-time Experiments}

\subsection{Setup}

The setup is composed of three Geomagic 3D Touch ${ }^{T M}$ haptic devices (see Figure 6). The devices' kinematic and dynamic parameters are available in. ${ }^{20}$ These devices are six d.o.f. systems but only three d.o.f. are actuated. Figure 5 shows the haptic device with the considered actuated degrees of freedom $\left(\theta_{1}, \theta_{2}\right.$ and $\left.\theta_{3}\right)$.

The control software was implemented in Matlab Simulink@ (see Figure 7). Concerning the software connection with the devices, the Open Haptics@ software library was used along with the Phantorque block introduced $\mathrm{in}^{1}$ and extended to simultaneously work with three devices. During all the tests, the real time clock is provided by this block. 


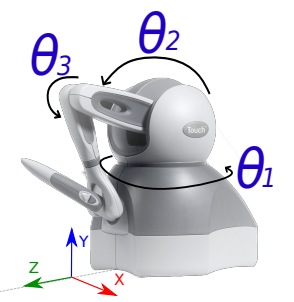

Fig. 5. The 3 controlled joints of the Geomagic $3 \mathrm{D}$ Touch $^{\mathrm{TM}}$ and the location of the user/cartesian frame

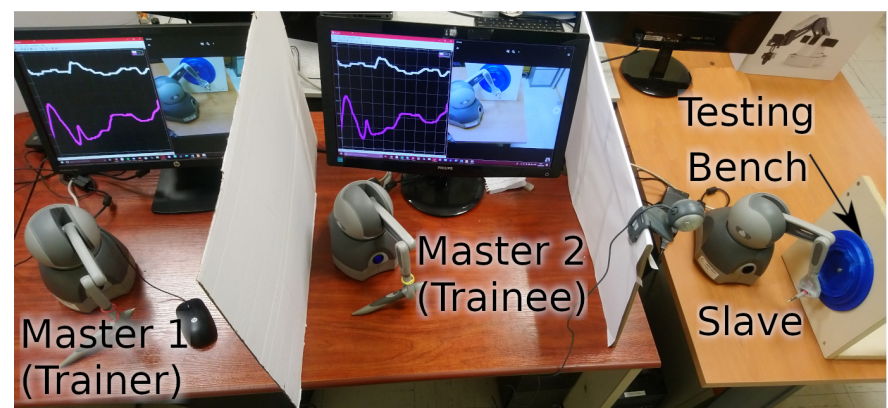

Fig. 6. Setup organisation

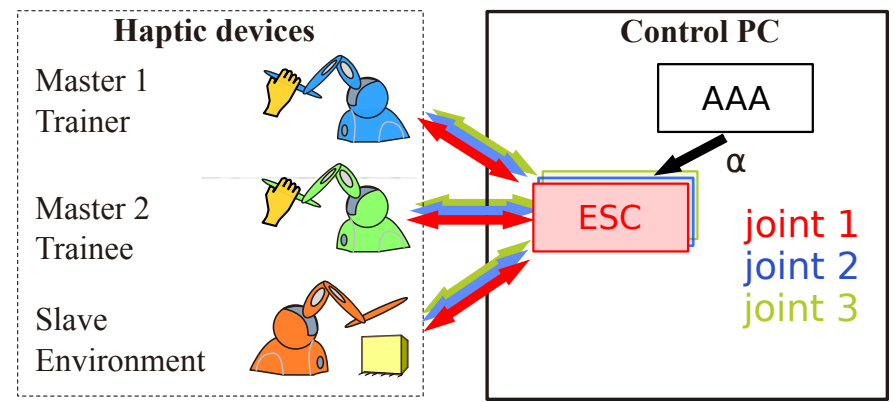

Fig. 7. Experimental architecture with an ESC for each joint and a global AAA

Table II . Real-time experiment parameters.

\begin{tabular}{llllllll}
\hline $\begin{array}{l}\text { Notation } \\
\text { Joints }\end{array}$ & Unit & \multicolumn{3}{c}{ Masters 1 and 2} & \multicolumn{3}{c}{ Slave } \\
\hline$m_{c}$ & & 1 & 2 & 3 & 1 & 2 & 3 \\
$k_{i_{m \mid s}}$ & $\mathrm{~kg} \cdot \mathrm{m}^{2}$ & $10^{-3}$ & $10^{-3}$ & $10^{-3}$ & $10^{-2}$ & $10^{-2}$ & $10^{-2}$ \\
$k_{i_{m}}$ & N.m $/ \mathrm{rad}$ & 15 & 10 & 10 & 35 & 20 & 10 \\
$b_{c_{m \mid s}}$ & N.m $/ \mathrm{rad}$ & 15 & 10 & 10 & & & \\
$b_{i_{m}}$ & N.m.s $/ \mathrm{rad}$ & $10^{-2}$ & $10^{-2}$ & $10^{-2}$ & 0.5 & 0.5 & 0.5 \\
\hline
\end{tabular}

The IPC controllers were tuned for compliant motion following the guideline provided in. ${ }^{24}$ Resulting parameters corresponding to the models introduced in the Appendix are visible in Table II.

\subsection{Free Motion}

In this first experiment, in order to show how mentoring and evaluation modes work, we set the dominance factor $\alpha=1$ at first and we suddenly changed it to 0 at $\mathrm{t}=25 \mathrm{~s}$ in order to determine how the system behaves on a sudden change of $\alpha$ Also, in order to show that the device of the following user effectively follows the one of the leader, each user performed a motion to take their device away from the trajectory prescribed by the 
leader, at their own turn. It it is visible in Figures 8, 9, and 10, for the trainee during mentoring stage (a) and for the trainer during evaluation stage (b).

Figure 8 depicts the global trajectory performed in the user/carterian space by the users in free motion. This plot shows that, out of the two attempts to take one of the devices away, they all haptic follow close trajectories. The root mean squared (RMS) value of the tracking error (computed from the positions expressed in the user/cartesian space) between the leading device (Master 1 when $\alpha=1$ and Master 2 when $\alpha=0$ ) and the slave was $1.8 \mathrm{~mm}$, for trajectories in a range of $18 \mathrm{~cm} \times 4 \mathrm{~cm} \times 10 \mathrm{~cm}$ corresponding to a volume of $72.10^{-5} \mathrm{~m}^{3}$. This level of precision corresponds to the one affordable with this type of off-the-shelf haptic device. The main limitation is the available maximum force around $3 \mathrm{~N}$. Some proposals to enhance the user experience are discussed later on.

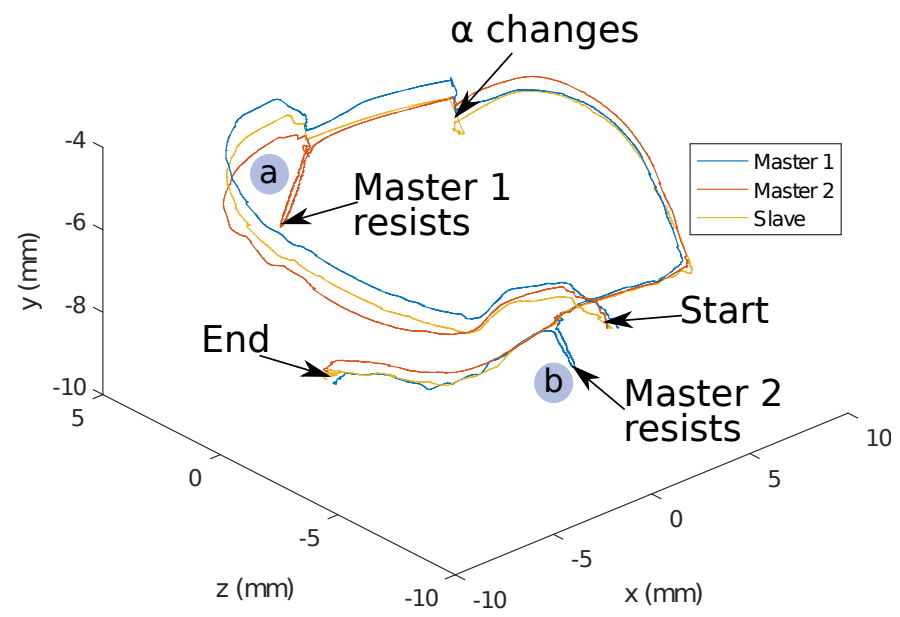

Fig. 8. Projection in space of the trajectory performed in free motion

The upper plots of Figures 9 and 10 show the positions of the three devices along $x$ and $y$ axis ( $z$ is not shown for reasons of space and the results are close to the other axis) in the user frame shown in Figure 5. They were computed from the angular positions $\Theta_{m 1}, \Theta_{m 2}$, and $\Theta_{s}$ of the three actuated joints, using the geometrical model provided in. ${ }^{1}$ In practice, these signals are directly available from inside the IPC controllers which exchange angular velocities with each other, for the dual-user operation needs (see Figure 2).

We obtained the best position tracking for $x$ (error less than $1 \mathrm{~mm}$ RMS between Master 1 and the slave when $\alpha=1)$ and the worst for $y(3.3 \mathrm{~mm} \mathrm{RMS})$. As $y$ is in the vertical direction, it highlights that the weight of the whole arm disturbs a little the IPC controllers. This controller was parameterized to provide the best transparency. Attempts to raise the gains of this joint controller led to oscillations and worst performance. A gravity compensation approach should be embedded to raise the precision in this direction.

Besides, the position tracking precision between the device of the following user and the slave is logically lower than between the leading user device, as the following user is guided by their device to follow the slave trajectory. According to the resistance they bring (the stiffness of their hand), the follower's position is shifted from the reference trajectory. The more the following users resist the motion of the device, the more the position tracking error raises (and so the force feedback) in order to bring them back to the leader's device position.

Lower plots of Figures 9 and 10 show the actuating forces of each device $F_{m 1}, F_{m 2}, F_{s}$ (in the user frame), computed from the torques $T_{m 1}, T_{m 2}, T_{s}$ applied on the three actuated joints, using the inverse dynamic model of the Geomagic 3D Touch ${ }^{\text {TM }}$ from. $^{1}$

These torques, generated by the IPC controllers for each actuated joint of each device are the only source of information about haptic feedback, as there is no force sensor measuring the real interaction forces in our setup. We must assume that the internal torque controller of the devices accurately applies them on each joint. This also 
14 An Energy-Based Approach for n-d.o.f. Passive Dual-user Haptic Training Systems

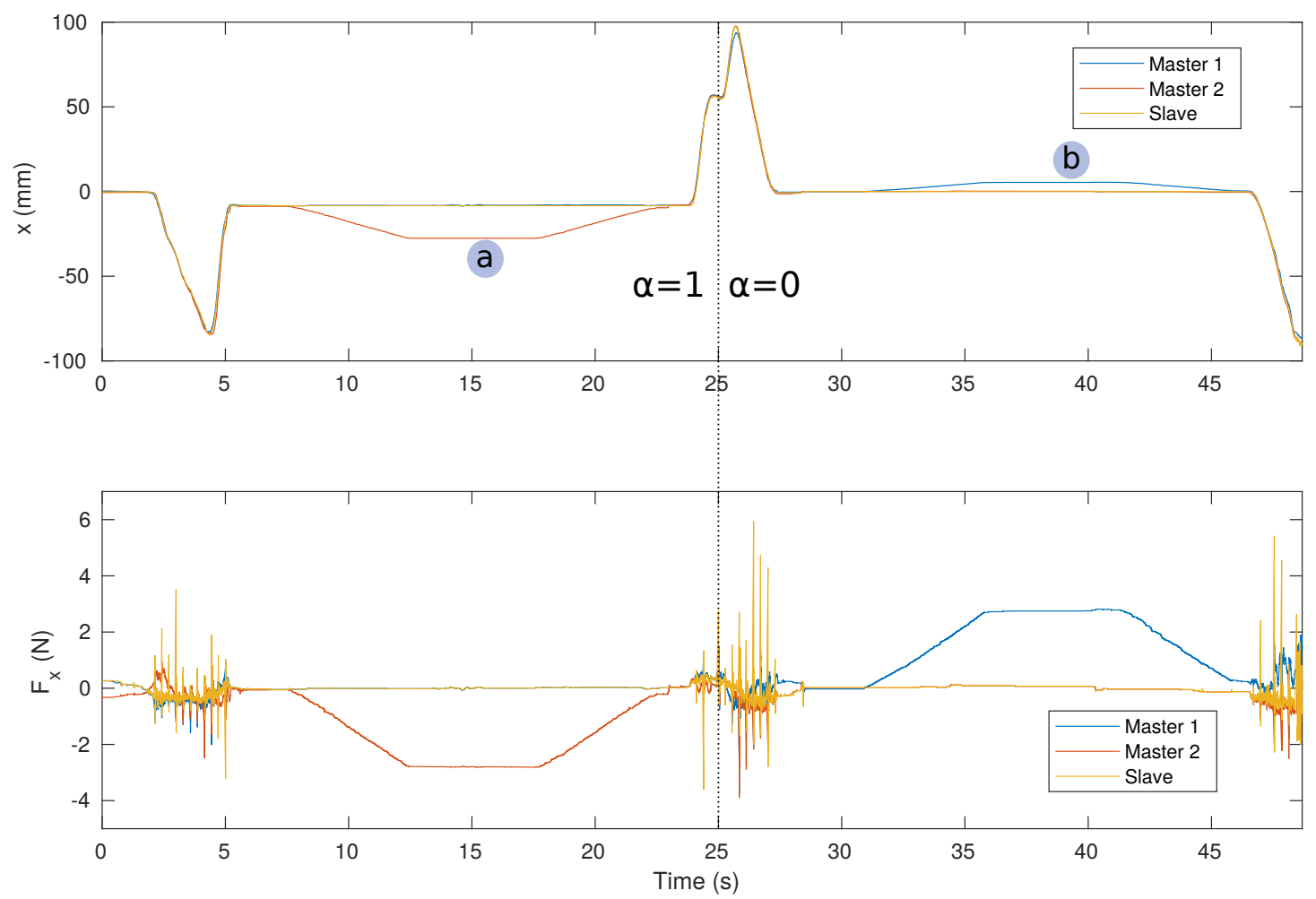

Fig. 9. Cartesian positions (upper plot) and forces (lower plot) for masters and slave in $x$ direction in the user frame in free motion

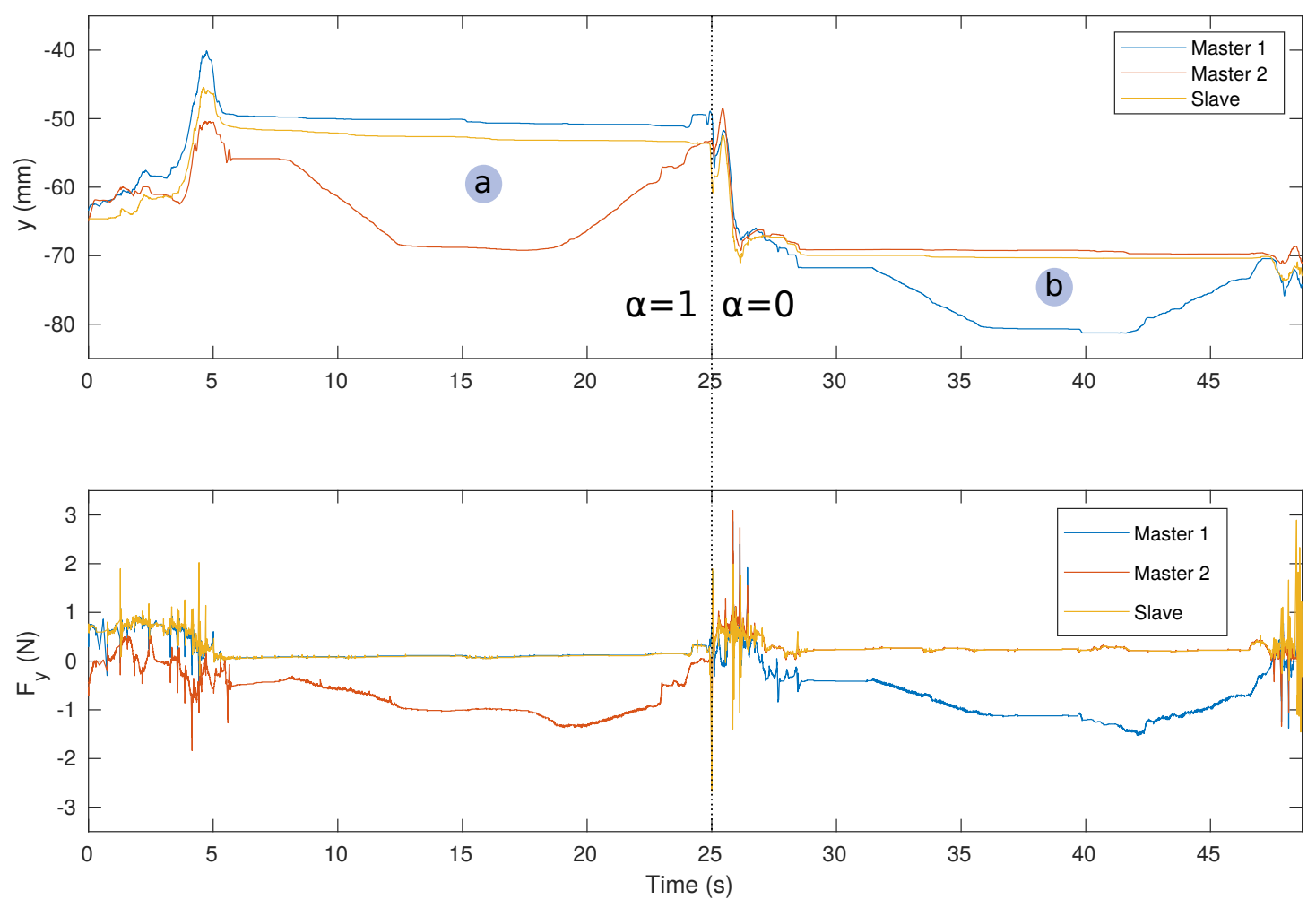

Fig. 10. Cartesian positions (upper plot) and forces (lower plot) for masters and slave in $y$ direction in the user frame in free motion 
means that these torques (which are also transmitted to the other devices) embed the (environment or user) interaction torques, the gravity effect and the friction torques. Therefore, the global system control is performed with these disturbed torques. Let notice that these torques are limited so that the resulting force is limited to $3.3 \mathrm{~N}$ in any direction of the cartesian space, according to the manufacturer. We took care to work below this threshold. In order to enhance the precision of the force measure, we should embed a real force sensor on each effector of these devices. Nevertheless, as the three devices feature the same equipment which have very close motions, these disturbances are, in practice, nearly the same for the three of them. Actually, they offset one another and the user only feels the feedbacks from tool-environment interaction. Also, the spikes visible in these plots (with some of them greater than $3 \mathrm{~N}$ ) when the devices are quickly moved are are not felt by users. We assume that the internal torque controller of the devices filters them. One can observe that, as the motion is performed in free motion, these forces are close to zero. Indeed, in mentoring (respectively evaluation) mode, the master 1 (respectively master 2) and slave forces overlap. Moreover, one can observe the forces generated by the devices to bring back the following user to the reference trajectory at marks (a) and (b). The sudden change of $\alpha$ has also no negative effect neither on forces nor on energy levels (see Figure 11) which remain negative.

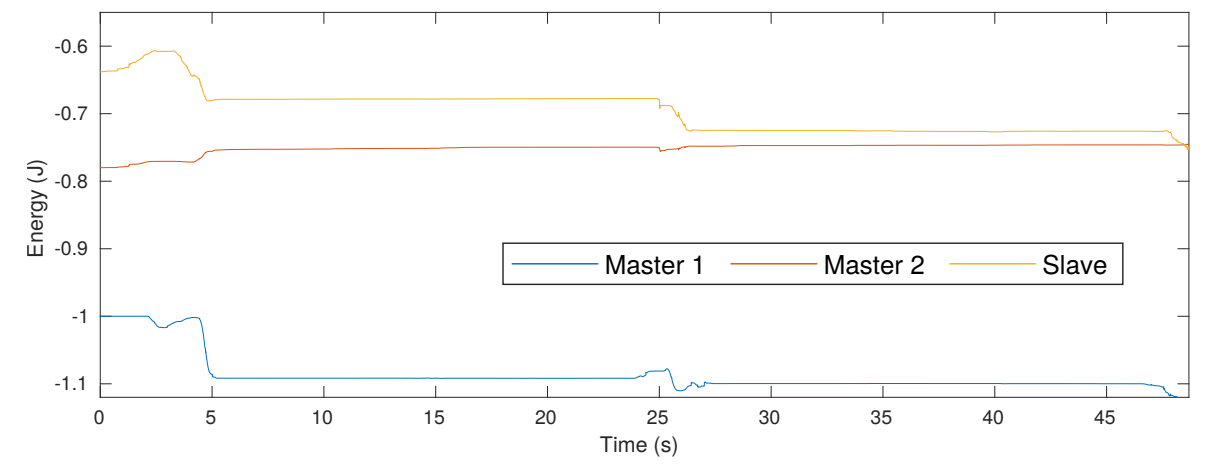

Fig. 11. Energy $E_{p}$ generated by both MFS for each joint in free motion

\subsection{In Contact Experiments}

In this second experiment, the dominance factor $\alpha$ is also changed from 1 to 0 , at $t=45 \mathrm{~s}$. The trainer leads their device to keep the slave effector on a surface which had the shape of a vertical circular guide slot (diameter of $133 \mathrm{~mm}$ ) and was rapid printed with ABS material (visible in Figure 6). This shape was chosen to illustrate a motion involving every joint. The surface is located in the plane $x=3 \mathrm{~mm}$ in the cartesian space. It was chosen so that the users push in the $x+$ direction to simplify the reading of the plots.

To evaluate the force feedback precision of the system, the leading user was assigned to keep their effector in contact while traveling in the circular slot. At two distinct instants, they had to stop their motion and to apply a higher push force, in order to ask the follower user to apply the same interaction force. This can be very useful in a training context to learn how to dose forces to apply. As any teleoperation system, bound to a balance between transparency and stability, the positions of the masters are virtually beyond the surface (around $18 \mathrm{~mm}$ ) while the slave effector stopped in contact, which corresponds to a stiffness of $130 \mathrm{~N} / \mathrm{m}$. We tried to increase the gains of the IPC controllers but it resulted in some annoying chattering. This stiffness could be raised by using more powerful off-the-shelf haptic devices (these ones are limited to $\approx 3 \mathrm{~N}$ in each direction).

Figure 12 shows the trajectory performed by the effectors. The spikes labeled with numbers from (1) to (4) correspond to the periods of time $(5<t<14 \mathrm{~s}, 20<t<40 \mathrm{~s}$, $45<t<60 \mathrm{~s}$, and $65<t<85 \mathrm{~s}$ ) when the leading users stopped their motion and pushed on the shape. It is clearly visible that the slave does not follow the leader master in the 


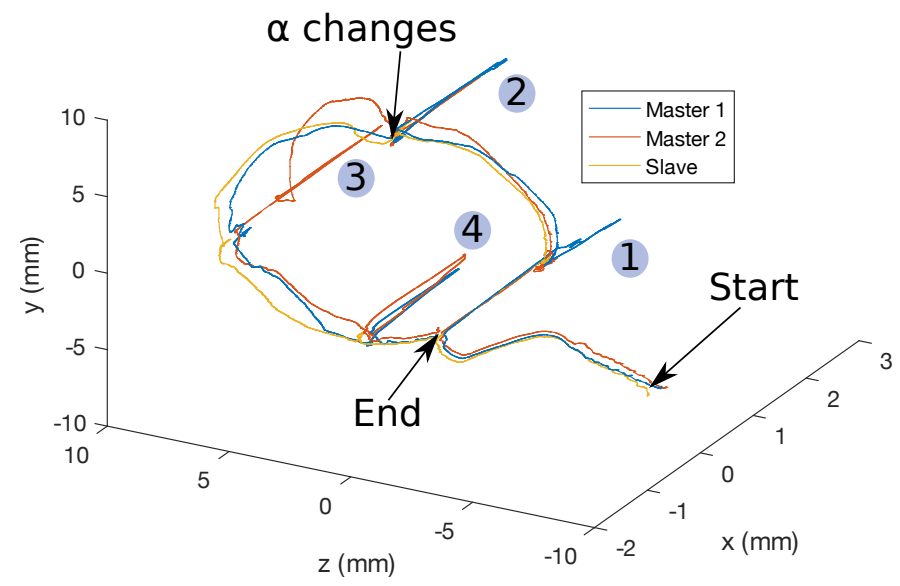

Fig. 12. Projection in space of the trajectory performed in contact case

$x+$ direction as it is in contact with the rigid surface. Only the masters go beyond this limit because of the aforementioned lack of stiffness.

Figure 14 shows the actuating forces of each device $F_{m 1}, F_{m 2}, F_{s}$ (in the user frame), as in previous free motion experiment. One can observe a good force tracking in the two dimensions $x$ and $y$ (the same for $z$ ). The more the leaders push their own device "inside the piece", the more the force feedback increases, as visible in Figure 14. In these plots, one can observe that the slave forces overlap or, at least, are very close to the leading master feedback interaction forces, which attests a good force tracking, and which is confirmed by the negligible level of the global force tracking error (computed from the magnitude of the difference of the forces in the three cartesian dimensions) between the slave and the leaders' forces: $0.2 \mathrm{~N}$ RMS, corresponding to $5 \%$ of the magnitude of the torques.

When the leaders decide this corresponds to the level of force they want the followers to replicate, they ask the followers to join them at the same (master) location. Its is done at $t=32 \mathrm{~s}$ and $t=75 \mathrm{~s}$. The followers align then their own device at the same location as the trainer's only by way of a video feedback and real-time plots displayed on their monitor, as visible in Figure 6 . For instance, at $t=38.5 \mathrm{~s}$, the trainee manages to have their own haptic device located at a distance of less than $1 \mathrm{~mm}$ in the $x$ direction. The interaction force in this direction is then at $2.4 \mathrm{~N}$, the trainer feels $2.5 \mathrm{~N}$ and the trainee $2.3 \mathrm{~N}$, which globally corresponds to an error of $4 \%$ compared to the slave force value. At $t=78 \mathrm{~s}$, the trainee and the slave are located at $x=17.2 \mathrm{~mm}$ while the trainer located their device at $x=16 \mathrm{~mm}$ (error of $1.2 \mathrm{~mm}$ : 7\%). The trainee feels the slave interaction force $(2.6 \mathrm{~N}$ with a precision better than $1 \%)$ while the trainer feels $2.4 \mathrm{~N}$, which corresponds to an error of $8 \%$.

This clearly shows that the better the following users position their device close to the same position as the leading user, the better the force feedback. On the one hand, a better alignment graphic tool should help minimize this error, and on the other hand, one has to take into account the Just Noticeable Difference (JND) of force that users can detect. In, ${ }^{18}$ a JND of $10 \%$ has been experimentally determined for forces detected by the index finger with forces from 2 to $5 \mathrm{~N}$. This corresponds to a JND force magnitude of $0.5 \mathrm{~N}$. Assuming it applies in our case, the force feedback errors measured in the experiments are below this level. They, therefore, should not be tangible by a human. More detailed experiments should be performed to confirm this assumption in our case.

Figure 15 clearly shows that MSF energy levels are not influenced by the contacts between the slave effector and its environment. 

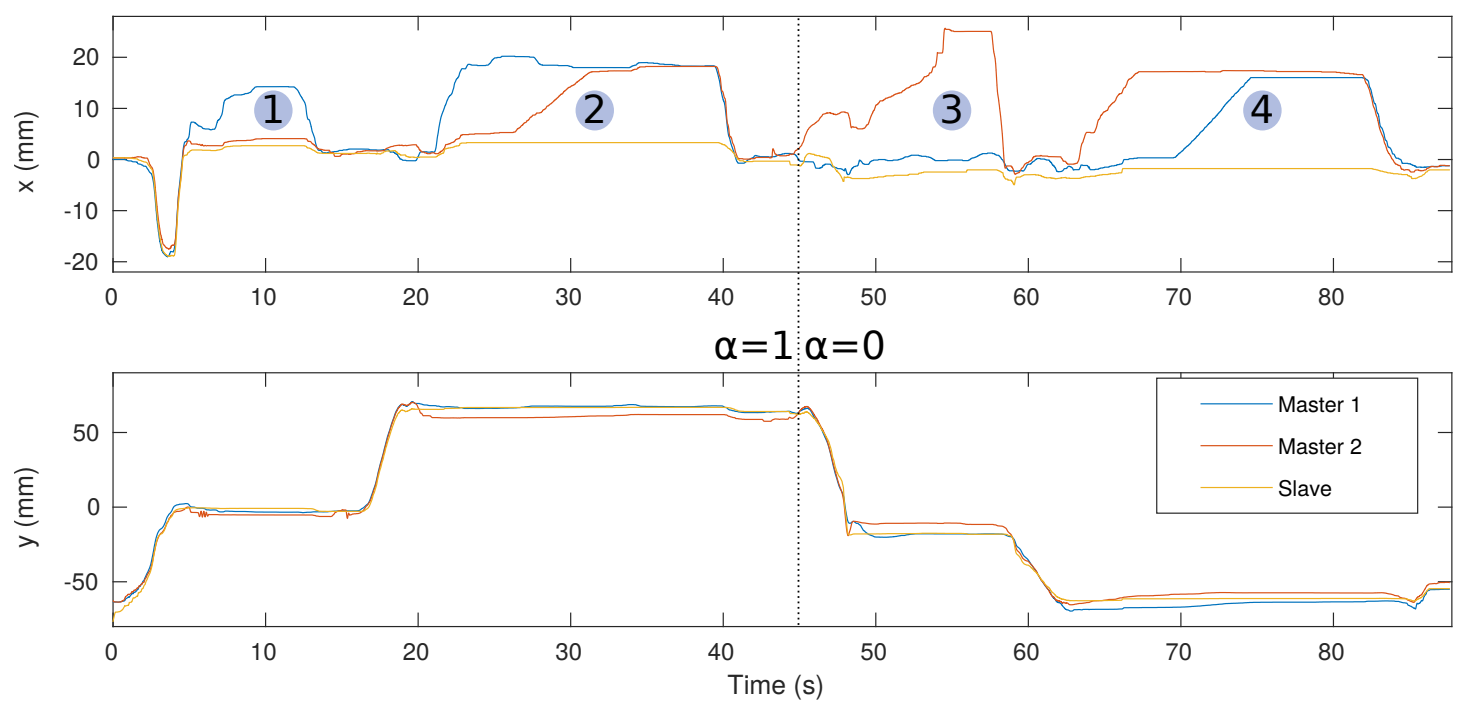

Fig. 13. Position for the trajectory performed in contact case in the cartesian space
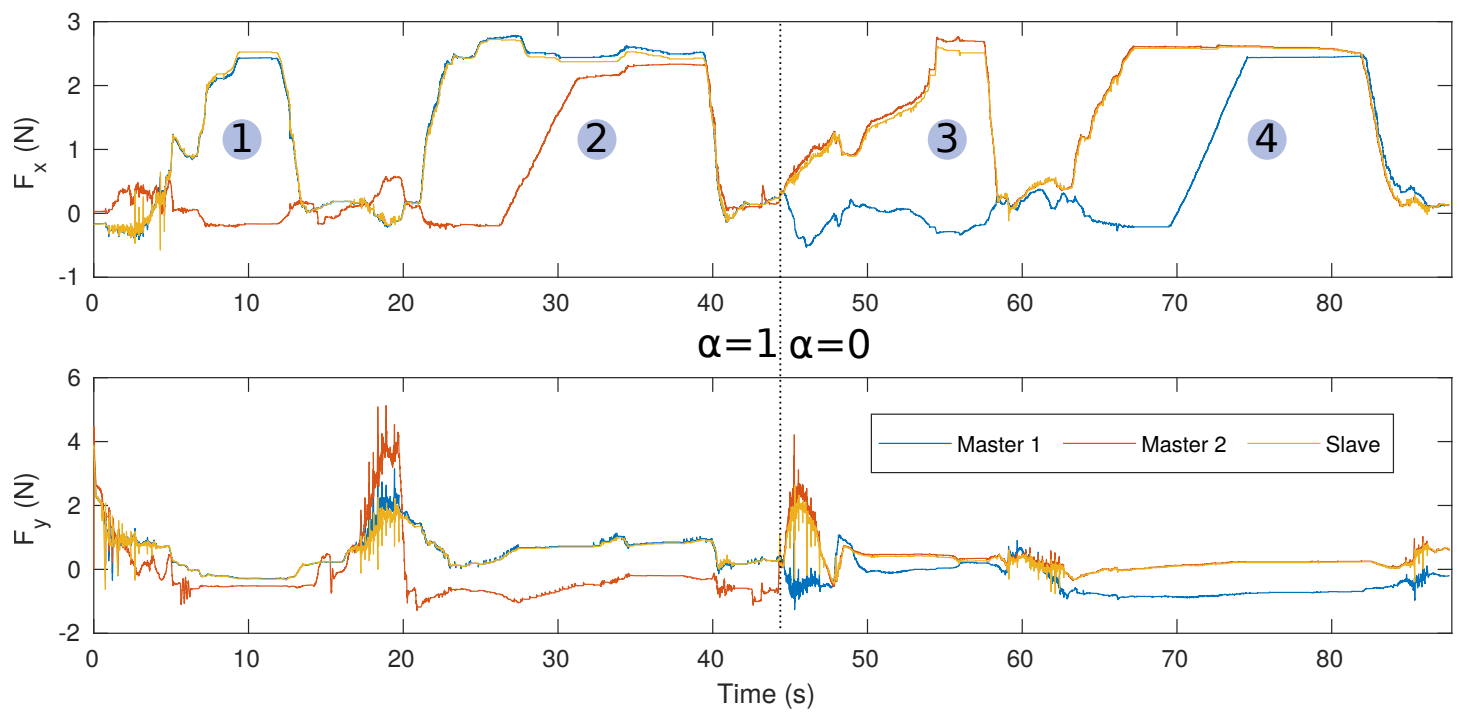

Fig. 14. Actuating forces for the three devices, expressed in the user frame, in contact case

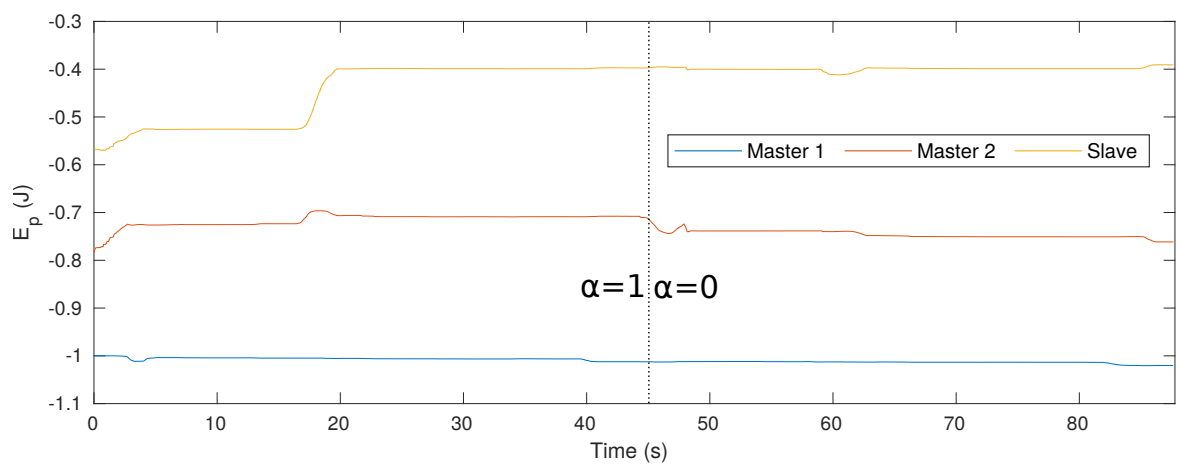

Fig. 15. Global Energy $E_{p}$ generated by both MFS for all the actuated joints, in contact case 


\subsection{AAA Validation}

Using the aforementioned AAA algorithm, an experimental validation has been performed to evaluate the behavior of the system. Some previous experiments took place only on a one d.o.f. system with the previous AAA algorithm. ${ }^{11}$ In Figure 18, the trajectories, in free motion, of the devices are shown in the user space with the corresponding force feedback. At first, $\alpha=0$. The trainee, by manipulating master 2 , guides the slave and the trainer (on master 1). At $t=5.6 \mathrm{~s}$, the trainer voluntarily progressively pulls away their device from the trainee's trajectory. As soon as the distance between the two master devices raises, $\alpha$ automatically grows and the trainer gets more control on the slave which progressively approaches the trainer's trajectory and then, when sufficiently far from the trainee's trajectory (at $t=10.6 \mathrm{~s}$ ), it fully follows them instead of the trainee: $\alpha$ grew up to 1 so that the trainer was full leader when $11<t<15 \mathrm{~s}$.

It can be observed that they get the force feedback from the slave during this period of time, in force plots of Figures 16 and 17.

When $0<\alpha<1$, the slave is in between both trajectories. It is sufficient to avoid, for instance, a mistake in the gesture of the trainee which could have damaged the tool or the environment. As soon as the trainee draws near the position of the trainer, $\alpha$ decreases so that the trainee can continue their exercise. In this experiment, the distance which raised $\alpha$ to 1 was $19 \mathrm{~mm}$. In practice, it should be adapted in real time by the trainer to the level of confidence in the skills of the trainee and according to the potential harmfulness of bad gestures.
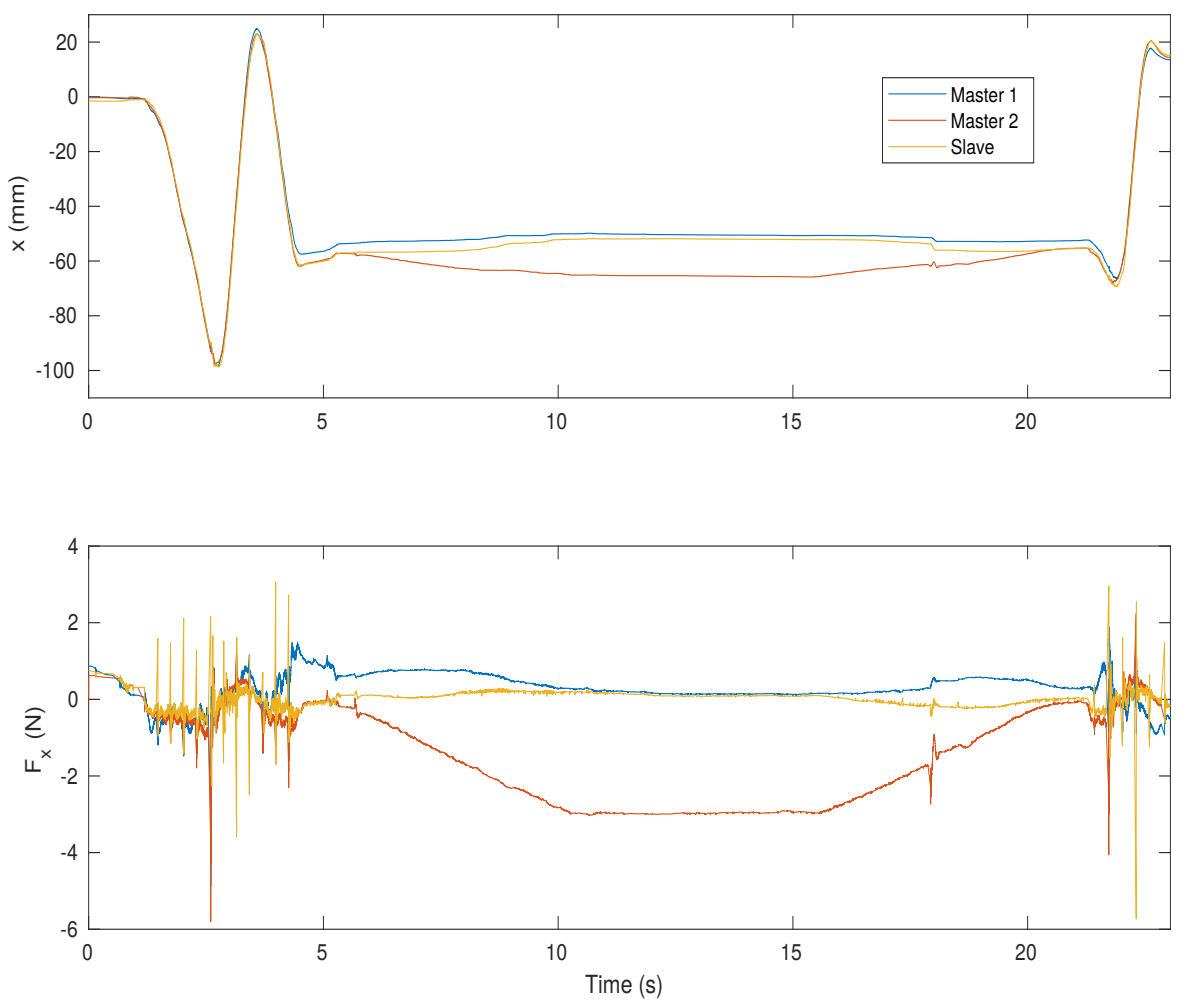

Fig. 16. Trajectories and feedback forces in $x$ direction in the user frame, with AAA function.

5.5. Conclusions about these experimental results

These experimental results demonstrate that the ESC architecture complies with the requirements evoked in the introduction. For a three d.o.f. system, the follower user is able to get the same force feedback as the leader when both users position their own 

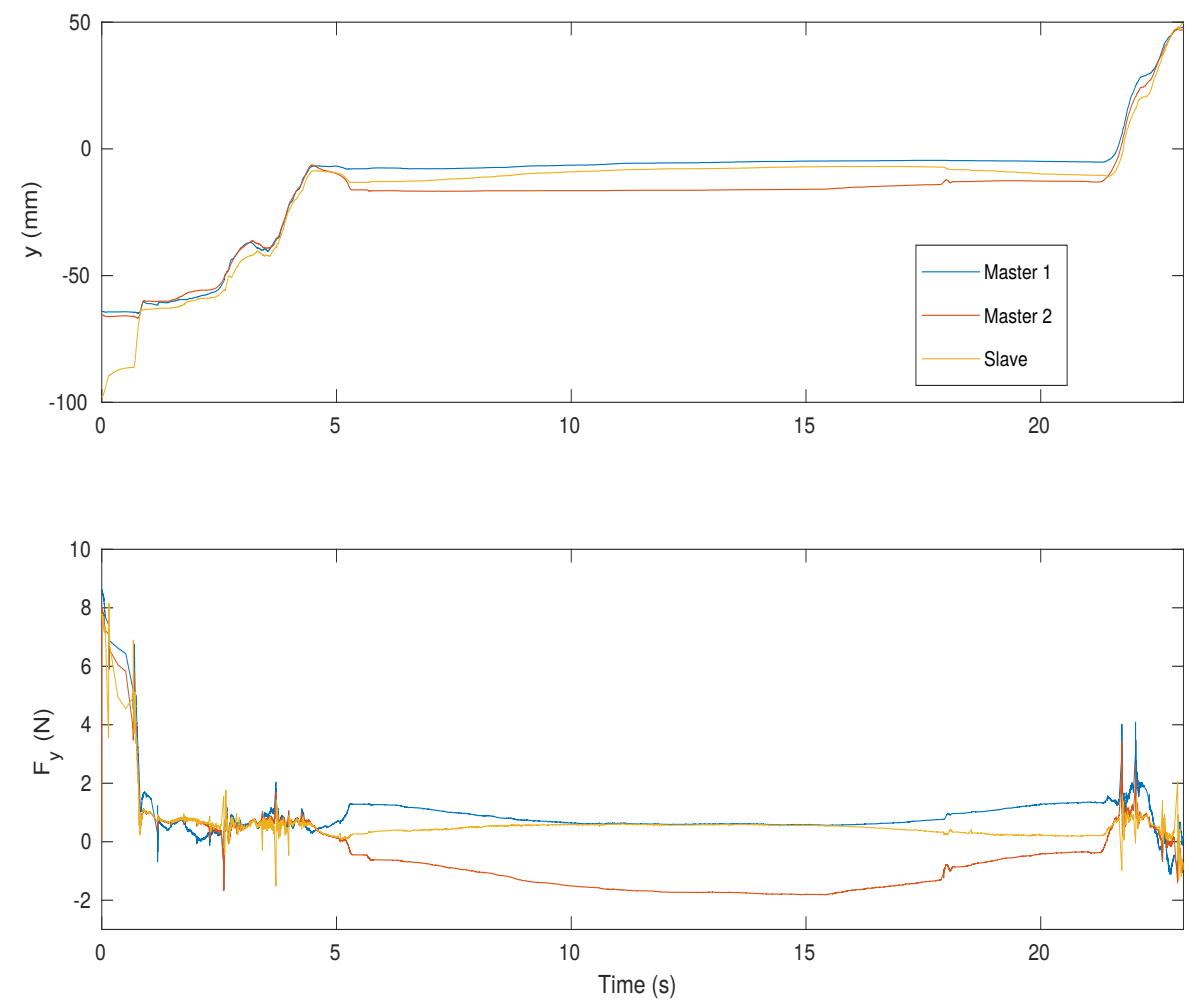

Fig. 17. AAA validation: trajectories and feedback forces in $y$ direction in the user frame, with AAA function.

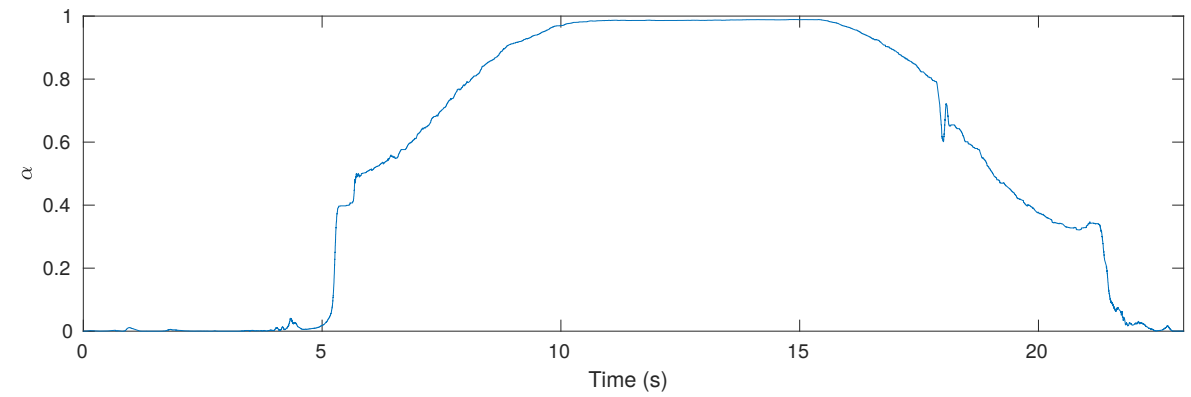

Fig. 18. AAA validation: evolution of $\alpha$

device at the same location. Also, the Adaptive Authority Adjustment mechanism proved it was efficient; it does not disturb the force feedback as long as the trainer follows (and, by the way, acknowledges) the trainee's motion. It permits the trainer to alternatively and transparently follow the trainee in evaluation mode, and to instantaneously take back the control of the slave to avoid any insecure situation. Experiments showed a global position tracking precision around $3 \mathrm{~mm}$ with a very good force tracking precision (less than $0.2 \mathrm{~N}$ ). We observed a larger tracking error in every situation in the $z$ axis which may be explained by the fact that, during the experiments, the arm is the most extended in this direction from the central vertical axis rotation. There may be a greater accumulation of sensor measure errors and model inaccuracy in this direction.

\section{Conclusion}

In order to provide a supervised hands-on training system, dual-user architectures suitable for such purpose have been surveyed. We proposed a typical use case which 
permitted to extract the requirements for such an activity and we observed which existing solutions could fit. As we found no solution responding to all these requirements, and considering the advantages of energy based modeling approaches, we extended in this paper the Energy Shared Control (ESC) dual-user architecture, from one to $n$ d.o.f., assuming each device is identical. . We detailed its modeling and motivated its architecture using the port-Hamiltonian framework. We also reported the extension to $n$ d.o.f. and the enhancement of the Automatic Authority Adjustment mechanism previously introduced in $^{11}$ for one d.o.f. The ESC performance has been validated experimentally: the results demonstrate a good slave position tracking performance (with a precision about $3 \mathrm{~mm}$ ) in both mentoring and evaluation mode and a full force feedback provided to both users in case of slave contact with environment with a precision around $0.2 \mathrm{~N}$. Experiments demonstrated that the AAA not only did not decrease this performance but it also facilitates the setting in real time of the authority, notably in emergency situations.

Nevertheless, the assumption that all devices share same kinematics may limit the applications. We will work on carrying this control architecture into cartesian space to open it to any combination of devices with different kinematics. We will also study a way to compensate for the gravity to enhance the positioning precision. Also, in this paper, we do not study the effect of delays in the loop as every device is supposed to be in the same room. The situation occurs when users are distant will be studied further, as in practice mentor surgeons work in hospitals while trainees study in their universities. A remote usage would be appreciated to gain travel time. Actually, it would be interesting to apply this architecture to a real supervised laboratory work and perform a comparative psychometric study to conclude definitely about the advantages of such an approach.

\section{Acknowledgements}

The authors acknowledge the financial support of the China Scholarship Council (CSC) and the Consejo Nacional de Ciencia y Tecnologia (CONACyT) in Mexico.

\section{Appendix}

\section{Modeling the Master/Slave Devices}

The dynamics of each $n$-d.o.f. haptic device $(d, i)^{2}$ with $i \in\left\{m_{1}, m_{2}, s\right\}$ (each master and the slave) can be modeled in the following port-Hamiltonian form with:

$$
\left\{\begin{array}{l}
\dot{X}_{d}=\left[J_{d}-R_{d}\left(X_{d}\right)\right] \frac{\partial H_{d}}{\partial X_{d}}+G_{d} U_{d} \\
Y_{d}=G_{d}^{T} \frac{\partial H_{d}}{\partial X_{d}}
\end{array}\right.
$$

where

$$
\begin{aligned}
J_{d} & =\left[\begin{array}{cc}
0 & I_{n} \\
-I_{n} & 0
\end{array}\right] \quad R_{d}=\left[\begin{array}{lc}
0 & 0 \\
0 & B_{d}\left(\Theta_{d}, P_{d}\right)
\end{array}\right] \\
G_{d} & =\left[\begin{array}{cc}
0 & 0 \\
I_{n} & I_{n}
\end{array}\right]
\end{aligned}
$$

\footnotetext{
2 for reasons of clarity, every subscript is written $d$ for "device" instead of $(d, i)$. Nevertheless, signal values are different between masters and slave
} 
and $X_{d}:=\left[\begin{array}{ll}\Theta_{d}^{T} & P_{d}^{T}\end{array}\right]^{T}$ is the state vector for each interface $\left(X_{d} \in \mathbb{R}^{2 m}, m \geq n\right) . \Theta_{d} \in \mathbb{R}^{n}$ is a vector of $n$ joint positions and $P_{i} \in \mathbb{R}^{n}$ a vector of $n$ joint momenta. $B_{d}\left(\Theta_{d}, P_{d}\right) \in$ $\mathbb{R}^{n \times n}$ is a symmetric positive-definite matrix, representing the dissipation elements.

The input $U_{d}$ and output $Y_{d}$ matrices are:

$$
U_{d}=\left[\begin{array}{c}
U_{d_{E}} \\
U_{d_{C}}
\end{array}\right]=\left[\begin{array}{c}
T_{\text {ext }_{d}} \\
T_{\text {des }_{d}}
\end{array}\right] \quad Y_{d}=\left[\begin{array}{c}
Y_{d_{E}} \\
Y_{d_{C}}
\end{array}\right]=\left[\begin{array}{c}
\dot{\Theta}_{d} \\
\dot{\Theta}_{d}
\end{array}\right]
$$

where $E$ and $C$ subscripts respectively indicate Environment and Controller sides. For the inputs, $T_{\text {des }_{d}} \in \mathbb{R}^{n}$ is the desired torque while $T_{\text {ext }} \in \mathbb{R}^{n}$ is an external torque exerted on the considered device.

The total energy of the device $H_{d}$ (Hamiltonian) is the sum of the global kinetic energy due to the inertial elements $M_{d}\left(\Theta_{d}\right) \in \mathbb{R}^{m \times m}$ and of a global potential energy $V_{d}\left(\theta_{d}\right)$ due to the gravity:

$$
H_{d}\left(X_{d}\right)=\frac{1}{2} P_{d}^{T} M_{d}^{-1}\left(\Theta_{d}\right) P_{d}+V_{d}\left(\Theta_{d}\right)
$$

IPC Controller

This section recalls the controller model used for each master, for a single d.o.f. It is an Intrinsically Passive Controller introduced by Stramigioli. ${ }^{25}$

Each master's IPC controller $(c, j), j \in\left\{m_{1}, m_{2}\right\}^{3}$ (see Figure 19) consists of a virtual mass, two springs and a damper The angular displacements of the two springs are represented by $q_{r_{m}} \in \mathbb{R}$ and $q_{c_{m}} \in \mathbb{R}$, respectively, and the momentum of the virtual mass by $p_{c_{m}} \in \mathbb{R}$. Each controller state variable is $x_{m}:=\left[\begin{array}{lll}q_{r_{m}} & p_{c_{m}} & q_{c_{m}}\end{array}\right]^{T}$ and its Hamiltonian is

$$
H_{m}\left(x_{m}\right)=\frac{1}{2} k_{r_{m}} q_{r_{m}}^{2}+\frac{1}{2} k_{c_{m}} q_{c_{m}}^{2}+\frac{p_{c_{m}}^{2}}{2 m_{m}}
$$

where $k_{r_{m}} \in \mathbb{R}$ and $k_{c_{m}} \in \mathbb{R}$ are the elasticity factors, and $m_{m} \in \mathbb{R}$ represents the inertial element.The port-Hamiltonian model of Master IPC is:

$$
\left\{\begin{array}{l}
\dot{x}_{m}=\left[J_{m}\left(x_{m}\right)-R_{m}\left(x_{m}\right)\right] \frac{\partial H_{m}}{\partial x_{m}}+G_{m} u_{m} \\
y_{m}=G_{m}^{T} \frac{\partial H_{m}}{\partial x_{m}}
\end{array}\right.
$$

where

$$
\begin{gathered}
J_{m}=\left[\begin{array}{ccc}
0 & -1 & 0 \\
1 & 0 & -1 \\
0 & 1 & 0
\end{array}\right] \quad R_{m}=\left[\begin{array}{ccc}
0 & 0 & 0 \\
0 & b_{c_{m}} & 0 \\
0 & 0 & 0
\end{array}\right] \\
G_{m}=\left[\begin{array}{ll}
0 & 1 \\
0 & 0 \\
1 & 0
\end{array}\right] \quad u_{m}=\left[\begin{array}{l}
\dot{\theta}_{m_{R}} \\
\dot{\theta}_{m_{N}}
\end{array}\right] \quad y_{m}=\left[\begin{array}{l}
t_{m_{R}} \\
t_{m_{N}}
\end{array}\right]
\end{gathered}
$$

where subscripts $R$ and $N$ respectively mean Robot and Network controller sides and $b_{c_{m}}$ is the dissipation element.

Correspondingly, for the slave IPC controller, one spring has been removed (in comparison with the master IPC controller), to ensure a causal interconnection, as shown

3 all the same, every subscript is written here $m$ for "master controller instead of $(c, j)$. 
22 An Energy-Based Approach for n-d.o.f. Passive Dual-user Haptic Training Systems

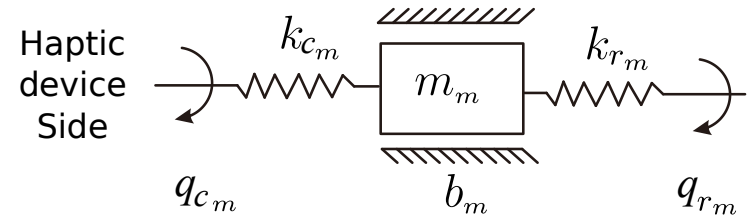

Fig. 19. Master IPC controller (in rotation context)

in Figure 20. The slave IPC controller, with a state vector $x_{s}:=\left[\begin{array}{ll}p_{s}^{T} & q_{s}^{T}\end{array}\right]^{T}$ has the following Hamiltonian:

$$
H_{s}\left(x_{s}\right)=\frac{1}{2} k_{i_{s}} q_{s}^{2}+\frac{p_{s}}{2 m_{s}}
$$

It is modeled in the following port-hamiltonian form:

$$
\left\{\begin{array}{l}
\dot{x}_{s}=\left[J_{s}\left(x_{s}\right)-R_{s}\left(x_{s}\right)\right] \frac{\partial H_{s}}{\partial x_{s}}+G_{s} u_{s} \\
y_{s}=G_{s}^{T} \frac{\partial H_{s}}{\partial x_{s}}
\end{array}\right.
$$

where

$$
\begin{aligned}
& J_{s}=\left[\begin{array}{cc}
0 & -1 \\
1 & 0
\end{array}\right] \quad R_{s}=\left[\begin{array}{cc}
b_{s} & 0 \\
0 & 0
\end{array}\right] \\
& G_{s}=\left[\begin{array}{ll}
0 & 1 \\
1 & 0
\end{array}\right] \quad u_{s}=\left[\begin{array}{l}
\dot{\theta}_{s_{R}} \\
t_{s_{N}}
\end{array}\right] \quad y_{s}=\left[\begin{array}{l}
t_{s_{R}} \\
\dot{\theta}_{s_{N}}
\end{array}\right]
\end{aligned}
$$

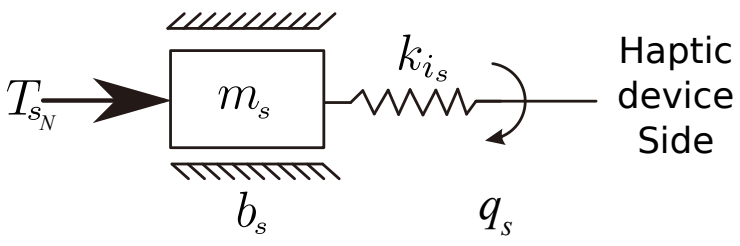

Fig. 20. Slave IPC controller

As the IPC controller parameters can be set arbitrarily without affecting its own (and the global) system passivity, one can tune them to get the best transparency from this system. The stiffness and damping parameters (in other words, the compliance) of each interface should be set so that the slave robot perfectly tracks the motion of the leader user and the users' interfaces feed back realistic environment force applied the on slave tool. Also, their damping should be set in order to avoid overshooting behaviors and undesirable oscillatory effects. A guideline for the IPC controllers is available. ${ }^{24}$

\section{References}

1. C. I. Aldana, E. Nuño, L. Basañez, and E. Romero. Operational space consensus of multiple heterogeneous robots without velocity measurements. Journal of the Franklin Institute, 351(3):15171539, 2014.

2. F. Beltran-Carbajal. Advances in Vibration Engineering and Structural Dynamics. InTech, 2012.

3. B. Chebbi, D. Lazaroff, and P. X. Liu. A collaborative virtual haptic environment for surgical training and tele-mentoring. Int. J. Robot. Autom., 22(1):69-78, Jan. 2007. 
4. S. Delorme, D. Laroche, R. DiRaddo, and R. F. Del Maestro. Neurotouch: A physics-based virtual simulator for cranial microneurosurgery training. Neurosurgery, 71:32-42, Sep. 2012.

5. V. Duindam, A. Macchelli, S. Stramigioli, and H. Bruyninckx. Modeling and Control of Complex Physical Systems: The Port-Hamiltonian Approach. Springer, 2009.

6. A. Ghorbanian, S. Rezaei, A. Khoogar, M. Zareinejad, and K. Baghestan. A novel control framework for nonlinear time-delayed dual-master/single-slave teleoperation. ISA Transactions, 52(2):268-277, Mar. 2013.

7. B. Khademian and K. Hashtrudi-Zaad. Shared control architectures for haptic training: performance and coupled stability analysis. The International Journal of Robotics Research, 30(13):1627-1642, Mar. 2011.

8. D. Lee and P. Li. Passive bilateral feedforward control of linear dynamically similar teleoperated manipulators. IEEE Transactions on Robotics and Automation, 19(3):443-456, Jun. 2003.

9. D. Lee and M. Spong. Passive bilateral teleoperation with constant time delay. IEEE Transactions on Robotics, 22(2):269-281, Apr. 2006.

10. A. R. Licona, F. Liu, A. Lelevé, D. Eberard, and M. T. Pham. Collaborative hands-on training on haptic simulators. Nov. 2018.

11. F. Liu, A. Lelevé, D. Eberard, and T. Redarce. A dual-user teleoperation system with adaptive authority adjustement for haptic training. In Proceedings of 4th International Workshop on Medical and Service Robots, Jul. 2015.

12. F. Liu, A. Lelevé, D. Eberard, and T. Redarce. A dual-user teleoperation system with online authority adjustment for haptic training. In Proceedings of the 37th Annual International Conference of the IEEE Engineering in Medicine and Biology Society (EMBC15), Milano, Italy, Aug. 2015.

13. F. Liu, A. Lelevé, D. Eberard, and T. Redarce. An energy based approach for passive dual-user haptic training systems. In Proceedings of the IEEE International Conference on Intelligent Robots and Systems (IROS 2016), Daejeon, South Corea, Oct. 2016.

14. Z. Lu, P. Huang, P. Dai, Z. Liu, and Z. Meng. Enhanced transparency dual-user shared control teleoperation architecture with multiple adaptive dominance factors. International Journal of Control, Automation and Systems, 15(5):2301-2312, Oct 2017.

15. E. Nuño, L. Basañez, and O. R. Passivity-based control for bilateral teleoperation: A tutorial. Automatica, 47(3):485-495, 2011.

16. S. Nudehi, R. Mukherjee, and M. Ghodoussi. A shared-control approach to haptic interface design for minimally invasive telesurgical training. IEEE Transactions on Control Systems Technology, 13(4):588-592, Jul. 2005.

17. L. Panait, E. Akkary, R. Bell, K. Roberts, S. Dudrick, and A. Duffy. The role of haptic feedback in laparoscopic simulation training. Journal of Surgical Research, 156(2):312-316, Oct. 2009.

18. M. S. Raghu Prasad, S. Purswani, and M. Manivannan. Force JND for right index finger using contra lateral force matching paradigm. In A. Chakrabarti and R. V. Prakash, editors, Proceedings of the Conference ICoRD'13, pages 365-375, India, 2013. Springer India.

19. K. Razi and K. Hashtrudi-Zaad. Analysis of coupled stability in multilateral dual-user teleoperation systems. IEEE Transactions on Robotics, 30(3):631-641, Jun. 2014.

20. T. Sansanayuth, I. Nilkhamhang, and K. Tungpimolrat. Teleoperation with inverse dynamics control for phantom omni haptic device. In Proceedings of SICE Annual Conference (SICE'12), pages 21212126, Aug. 2012.

21. M. Shahbazi, S. Atashzar, H. Talebi, and R. Patel. An expertise-oriented training framework for robotics-assisted surgery. In Robotics and Automation (ICRA), 2014 IEEE International Conference on, pages 5902-5907, May 2014.

22. M. Shahbazi, H. Talebi, S. Atashzar, F. Towhidkhah, R. Patel, and S. Shojaei. A new set of desired objectives for dual-user systems in the presence of unknown communication delay. In Advanced Intelligent Mechatronics (AIM), 2011 IEEE/ASME International Conference on, pages 146-151, July 2011.

23. K. Shamaei, L. Kim, and A. Okamura. Design and evaluation of a trilateral shared-control architecture for teleoperated training robots. In Engineering in Medicine and Biology Society (EMBC), 2015 37th Annual International Conference of the IEEE, pages 4887-4893, Aug 2015.

24. S. Stramigioli. Creating artificial damping by means of damping injection. In Proceedings of the ASME Dynamic Systems and Control Division, pages 601-606, 1996.

25. S. Stramigioli. Modeling and IPC control of interactive mechanical systems: A coordinate-free approach. Springer, 2001.

26. A. Talasaz, A. L. Trejos, and R. V. Patel. The role of direct and visual force feedback in suturing using a 7-dof dual-arm teleoperated system. IEEE Transactions on Haptics, 10(2):276-287, April 2017.

27. A. van der Schaft and D. Jeltsema. Port-hamiltonian systems theory: An introductory overview. Foundations and Trends in Systems and Control, 1(2-3):173-378, June 2014.

28. N. Vaughan, V. N. Dubey, M. Y. Wee, and R. Isaacs. A review of epidural simulators: Where are we today? Medical Engineering $\&$ Physics, 35(9):1235-1250, Sept 2013.

29. E. Yiannakopoulou, N. Nikiteas, D. Perrea, and C. Tsigris. Virtual reality simulators and training in laparoscopic surgery. International Journal of Surgery, 13(9):60-64, Feb. 2014.

30. A. Zakerimanesh, F. Hashemzadeh, and A. R. Ghiasi. Dual-user nonlinear teleoperation subjected to varying time delay and bounded inputs. ISA Transactions, 68:33-47, 2017. 